\title{
Choosing the Appropriate Amount of Mortgage Loan: Risk Based Decision Making
}

\author{
Suleyman Gokhan Gunay ${ }^{1} \&$ Kasırga Yıldırak ${ }^{2}$ \\ ${ }^{1}$ Faculty of Economics and Administrative Sciences,Trakya University, Edirne, Turkey \\ ${ }^{2}$ Department of Actuarial Science, Hacettepe University, Ankara, Turkey \\ Correspondence: Suleyman Gokhan Gunay, Faculty of Economics and Administrative Sciences, Trakya \\ University, Edirne, Turkey. Tel: 90-284-235-7152. E-mail: suleymangokhan@ gmail.com
}

Received: August 22, 2016

Accepted: September 26, 2016

Online Published: October 25, 2016

doi:10.5539/ijef.v8n11p12

URL: http://dx.doi.org/10.5539/ijef.v8n11p12

\begin{abstract}
The main purpose of this paper is to show the way to conduct a risk based mortgage loan choice process for low and middle income households in Turkey. There are several studies that analyze the impact of buying a house decision on an investor's portfolio, which consists of house, stocks and bonds. In this study, house is treated as a single investment. The probability of defaults for the cash flows based on mortgage payments, rents, down payment and depreciation are calculated in order to find the amount of mortgage loan for a given rent and maturity. Net present values of cash flows related with buying a house are calculated via stochastic interest rates based on historical simulation. It is found that the amount of mortgage loan can be determined by risk based approach and a rational choice can be made in terms of household risk management.
\end{abstract}

Keywords: mortgage loan, historical simulation, Nelson-Siegel method, probability of default, cash flows

\section{Introduction}

Interest rates have been declining in Turkey since 2001. This trend in interest rates on deposits can be seen in Figure 1 . The weighted average of interest rate for housing loans was approximately $54,84 \%$ on January 2002 . It is $13,31 \%$ on August 2016. The trend about the aggregate demand for the housing loans between 2005 and 2014 can also be seen in Figure 2. The aggregate demand for the housing loans was approximately 12 billion Turkish Lira (approximately 8,9 billion US dollars) on December 2005. It is approximately 105 billion Turkish Lira (approximately 49 billion US dollars) on June 2014. The yearly average increase in the demand for housing loans between these dates is about $91 \%$. Mortgage loans are first given by financial institutions in Turkey in 2006. The housing loans availability may ocur due to the abundancy of cash flows in the last decade. This sharp decline on interest rates and credit availability made it possible for many households to buy their own houses. Thus, the demand for the housing loans increased sharply in the last decade as a result of this trend in the housing finance market. 


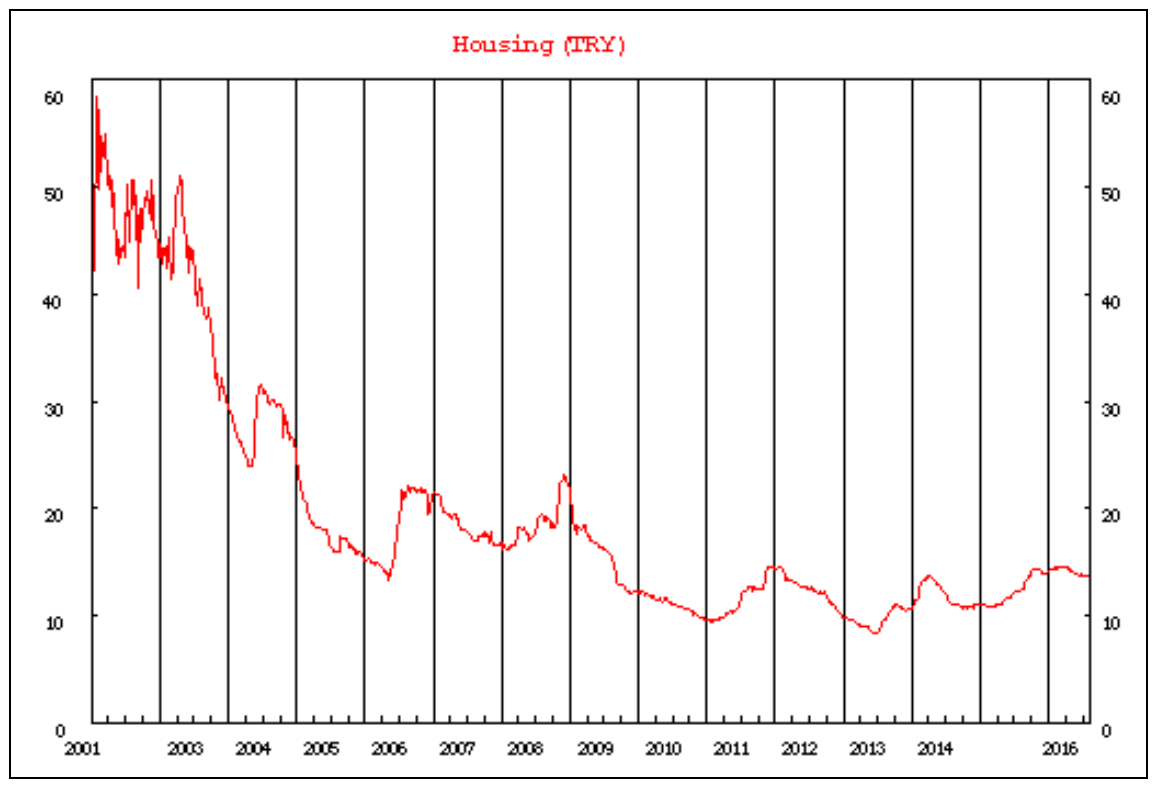

Figure 1. Weighted average interest rates for banks' housing loans between 2001 and 2016 Source: Central Bank of Turkey, Electronic Data Delivery System.

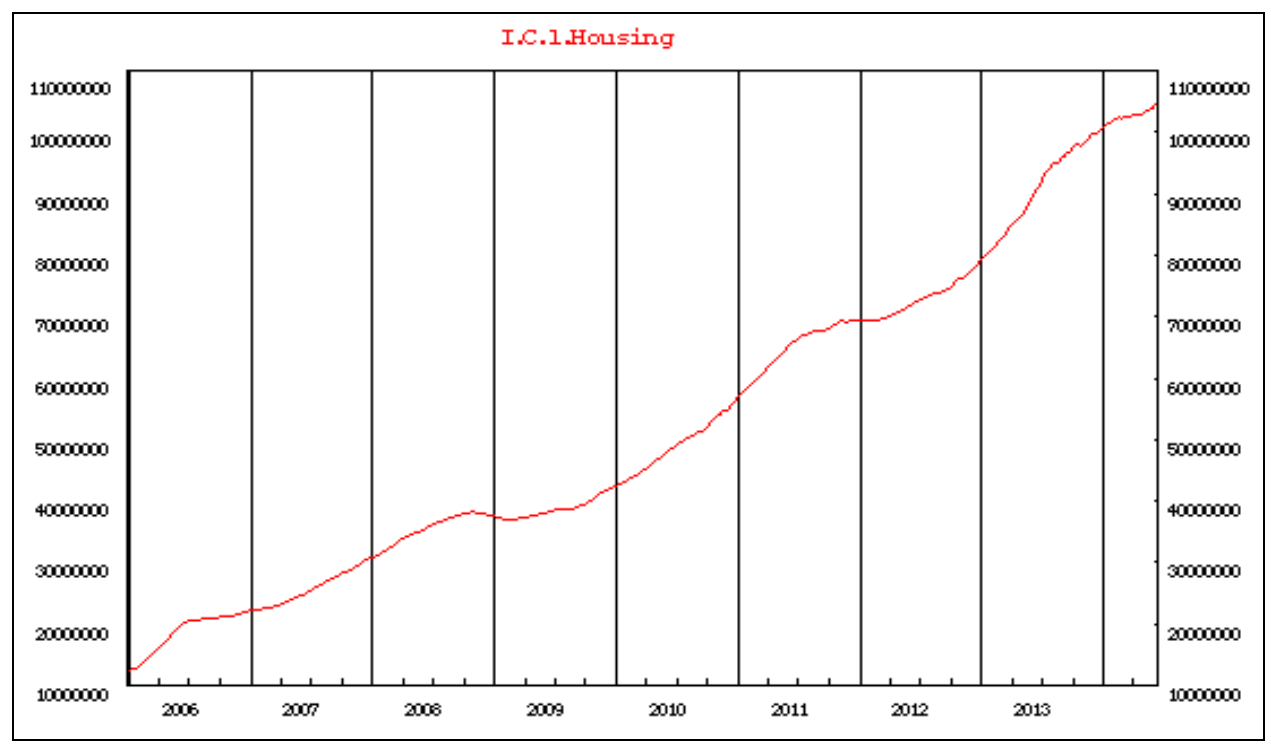

Figure 2. The aggregate demand for housing loans between 2005 and 2014 in Turkey

Source: Central Bank of Turkey, Electronic Data Delivery System.

Lower mortgage loan monthly payments motivated most of the households to buy their own houses in Turkey due to the sharp decline in interest rates. But the households do not necessarily always make financial decisions based on risk assessment before buying their houses. In other words, the risk adjusted appropriate level of mortgage loan that will make households better-off is not known by these economic actors. This is an exploratory research, which uses empirical facts to help individuals to act in a risk oriented manner in their decisions in the process of buying their houses.

Literature review is presented in the second section of the paper. Cash flow analysis based on net present value is given in the third section. Yield curve based on zero-coupon bonds are embedded to the model in order to discount the cash flows in the analysis. Cash flows analysis related with housing stock investment is based on historical simulation method. Comparison and advantages of Nelson-Siegel method with alternative 
methodologies are given in the fourth section. The findings of this study is presented in the fifth section. Finally, conclusion is given in the sixth section of the paper.

\section{Literature Review}

Households can meet their housing need in two different ways: they can rent housing services, or they can purchase housing stocks. When the price of renting positively correlates with house prices, home-ownership is a way to reduce the risk, related to the consumption of housing services (Sinai \& Souleles, 2005). A decision must be made between buying and renting a house. The purpose of our paper is to help households in Turkey to make this important decision rationally. Thus, a house is treated as a financial investment decision in this study. Housing stock is treated as an asset by many scholars (Elton \& Gruber, 1992; Flavin \& Yamashita, 2002; Goetzmann \& Ibbotson, 1990; Goetzmann, 1993; Pelizzon \& Weber, 2008; Ross \& Zisler, 1991). The problem of choosing an optimal mortgage contract is a complex one. Households must take into consideration real interest rate risk, inflation risk, borrowing constraints today and the possibility of borrowing constraints in the future, their risk aversion, their moving probability, and their ability to refinance a fixed-rate mortgage optimally (Campbell \& Cocco, 2003; Ehrmann \& Ziegelmeyer, 2014).

Several academics (Chetty \& Szeidl, 2010; Cocco, 2005; Cocco, Gomes, \& Maenhout, 2005; Corradin, Fillat, \& Vergara-Alert, 2012; Fisher \& Goetzmann, 2005; Flavin \& Yamashita, 1997; Grossman \& Laroque, 1990; Heaton \& Lucas, 2000; Yao \& Zhang, 2005; Zoutman, 2015) analyze the impact of a housing decision on an investor's portfolio, which consists of house, stocks and bonds. Our study differs from the previous studies in several important dimensions. First, we treat a house as a single investment. Second, we compute the probability of defaults caused by interest risk given different levels of mortgage payments, rents, down payments and depreciation rates in this study. Third, we show the way how to make a risk based rent-mortgage contract decision.

\section{Cash Flow Analysis Based on Net Present Value}

We combine the cash flows generated from the value of the house, rent income, mortgage loan payments and the value of down payment as a single entity and compute the net present value of the related cash flows. Rents and the salvage value of a house are treated as cash inflows in the analysis, while the value of monthly payments of a mortgage loan are considered as cash outflows. The salvage value of a house at the end of a mortgage loan is calculated by deducting the depreciation of the house during the duration of the mortgage loan. Since down payment in a mortgage loan can be depositted to a bank, it is also treated as cash outflow in the analysis in the sense of opportunity cost. Loan to Value (LTV) ratio is $\% 75$ for the mortgage loans in Turkey. Since a sharp increase in the interest rates can be seen due to an economic shock in the future, in our cash flow analysis, adjustable-rate mortgage (ARM) is perceived as a gamble, which is also advocated by Fisher and Shelly (2002). Thus, mortgage loans are treated as fixed-rate mortgage (FRM) through the study.

The relation between maturity and the depreciation rate $(\delta)$ is expressed as:

$$
\delta=\left[\frac{1}{2}\left(\frac{P_{a}^{2}}{P_{e}^{2}}+\frac{P_{a}}{P_{e}}\right)\right] * 100
$$

Where $\mathrm{P}_{\mathrm{a}}$ represents actual age of property and $\mathrm{P}_{\mathrm{e}}$ represents the effective age of property. $\delta$ is the depreciation rate on the property. Effective age of a house is determined as fifty years. Interest risk is analyzed under different scenarios namely for different maturities and depreciation rate, and also for different rent scheme. Maturities and their corresponding depreciation rates are calculated as follows:

\begin{tabular}{|l|c|c|c|c|c|c|c|c|c|c|c|}
\hline Maturity & 10 & 11 & 12 & 13 & 14 & 15 & 16 & 17 & 18 & 19 & 20 \\
\hline Depreciation & 0.1200 & 0.1342 & 0.1488 & 0.1632 & 0.1798 & 0.1950 & 0.2112 & 0.2278 & 0.2448 & 0.2622 & 0.2800 \\
\hline
\end{tabular}

Monthly mortgage payments are calculated based on the annuity formula below:

$$
\text { Mortgage Loan }=M P * \frac{(1+i)^{n}-1}{(1+i)^{n} * i}
$$

Where MP is the monthly mortgage payments, $i$ is the fixed interest rate of the mortgage loan, and $n$ is the number of mortgage payments in the equation above.

Total value of the mortgage payments could be broken down as follows: 


$$
\text { Total Rent Income }(\text { TRI })=\sum_{i=1}^{N} 12 * R *\left(1+\pi_{i}\right)
$$

Where $R$ and $\pi_{i}$ represent the initial value of the rent and the annual inflation rate respectively. $N$ is the years to maturity.

$$
\text { Value of the Property }(V P)=\left(H * \prod_{i=1}^{N}\left(1+\pi_{i}\right)\right) *(1-\delta)
$$

where $H$ is the initial value of the property. Then formula for the net present value of the cash flows (NPVCF) related with rents, value of property, monthly mortgage payments and down payment is as follows:

$$
N P V C F=\sum_{i=0}^{N-1}\left(R \prod_{m=0}^{i}\left(1+\pi_{m}\right) \sum_{j=i * 12+1}^{i * 12+12} e^{-r_{j}}\right)+V P e^{-r_{T}}-\sum_{k=1}^{12 * N} M P_{k} e^{-r_{k}}-\sum_{l=1}^{12 * N} D P_{l} e^{-r_{l-1}}
$$

Where $D P_{l}=D P *\left(1+r_{b}\right)^{l}$, which is the value of the down payment at period $l . r_{b}$ is the rate of return on bank account, $r$ is the yield on Turkish treasury bonds, and $T$ is the maturity date of the mortgage contract.

\section{Nelson-Siegel Yield Curve Construction and Historical Simulation Method}

The commonly used term structure estimation methods are given as the bootstrapping method, the polynomial/exponential spline methods of McCulloch $(1971,1975)$ and Vasicek and Fong (1982), and the exponential functional form methods of Nelson and Siegel (1987) and Svensson (1994). Nelson and Siegel (1987) proposed to fit the term structure using a flexible, smooth parametric function. This model is capable of capturing many of the typically observed shapes that the yield curve assumes over time and it is used to construct zero-coupon yield curves. Besides, Nelson and Siegel method is more robust than other methods. Moreover, in this study, our interest is to measure the risk of a mortgage loan which naturally is subject to a long term interest rate risk. Thus, by definition, Nelson and Siegel method will be a superior choice over spline based methods.

Let the forward rate follows the following equation:

$$
f(\tau)=\beta_{0}+\left(\beta_{1}+\beta_{2} \tau\right) \exp \left(-\beta_{3} \tau\right)
$$

Where, $\beta_{0}$ is the long rate, $\beta_{0}+\beta_{1}$ is the short rate and $\tau$ is the time to maturity. Then the spot rate is expressed as:

$$
r(\tau)=\beta_{0}+\left(\beta_{1}+\beta_{2}\right)\left(1-\exp \left(-\frac{\tau}{\beta_{3}}\right)\right) \frac{\beta_{3}}{\tau}-\beta_{2} \exp \left(-\frac{\tau}{\beta_{3}}\right)
$$

Estimation of the parameters $\Theta=\left\{\beta_{0}, \beta_{1}, \beta_{2}, \beta_{3}\right\}$ is done by restricted nonlinear least squares method. Objective function for the optimization is:

$$
\min _{\Theta}\left(P^{N S}(r(\tau))-P\right)^{\prime} w^{\prime} w\left(P^{N S}(r(\tau))-P\right)
$$

Where $P^{N S}(r(\tau))$ is the price of the bond with maturity $\tau, \mathrm{P}$ is the observed price and $\mathrm{w}$ is the weight vector of modified duration of corresponding bonds.

Recently, Diebold and Li (2006) and Diebold, Rudebusch, and Aruoba (2006) have shown that the three-factor Nelson-Siegel model can also be used to construct accurate term structure forecasts. By using a straightforward two-step estimation procedure, they demonstrate that Nelson-Siegel model performs well when compared with the competing models, especially for longer forecast horizons. These results are partially confirmed by Mönch (2006). Nelson-Siegel model produces forecasts that are not only statistically accurate but also economically meaningful (Fabozzi, Martellini, \& Priaulet, 2005). Due to these successes it is not surprising that the Nelson-Siegel model is increasingly being used in other applications as well. For example, Diebold, Piazzesi, and Rudebusch (2005) and Diebold, Rudebusch, and Aruoba (2006) use the model to study the interactions between the macro economy and the yield curve. Diebold, Ji, and Li (2006) apply it to identify systematic risk sources and to construct a generalized duration measure. De Pooter (2007) also has shown that the four-factor Nelson-Siegel model can also be used to construct accurate term structure forecasts. Tsui, Wu and Zhang (2013) used compared Nelson-Siegel model with the non-Nelson-Siegel models in forcasting the yield curve in Japan by using the data from Januar 2000 to November 2007. Molenaars, Reineirnk, and Hemminga (2015) used the model to test the random walk hypothesis by using the data from 1971 to 2008. 
Interest risk of the cash flows is computed by historical simulation. Steps followed for the simulation are as the following:

1) Construct the daily yield curves with one to $\mathrm{T}$ days to maturity by Nelson-Siegel method for $\mathrm{N}$ days.

2) Take the 360-day difference of the data and save it in a vector of (N-360) by $\mathrm{T}$.

3) Add the saved vector to the last day yield observations and construct the simulated vectors of yields.

4) Compute $\mathrm{N}-360$ of net present value of the future mortgage payments.

5) Count how many times the negative net present values obtained and divided by N-360 and report this number as the default frequency caused by the variation in interest rate.

Historical simulation is criticized for not being able to reflect the probability of the events not experienced within the data set. For this reason, parametric, semi parametric and Monte Carlo simulation based risk estimation methods are suggested for practitioners. However, for the realized interest rate process in Turkey, it would be very hard to find a proper theoretical stochastic process and probability distribution. In addition to difficulty of proper model choice, computation burden would be higher than in historical simulation. Moreover, our main purpose is to build a tool to compare the risk of various mortgage action scenarios, not to compute the exact default probabilities.

\section{Risk Based Mortgage Loan Choice for a Given Value of Rent, Maturity of the Loan and Interest Rates}

The level of mortgage loans is determined by three factors. The first factor is the rent. Rent varies between zero and 1,000 T.L. These are the minimum and maximum amounts of rent that are used in the analysis based on the economic conditions in Turkey. If the landlord cannot rent his or her house, the rent may be zero until the end of the mortgage loan. Even if a person buys a house for himself or herself, there is still opportunity cost of not paying a rent to another house because of making this investment. The second factor is the amount of mortgage loans. This factor is shown with the term "Amount" in Figure 3. The third factor is the interest rate. As it is mentioned in the previous section, the influence of interest rates via yield curve is embedded to analysis by using historical simulation. There is another factor which depends on these three factors. This factor is the probability of default (PD). It shows the probability of having a negative net present value in an one year holding period.

As can be seen in Figure 3, there are four kinds of colours. These colours show the risk levels for a certain amount of mortgage loan, rent, and maturity. The area shown with dark blue is the zero-risk area. For example, the area shown with the letters ABK is the zero-risk area for mortgage loans. In other words, ABK shows area for mortgage loans with zero risk. The area shown with red shows the mortgage loans with the highest risk. Layers in Figure 3 shows the maturity, which is between ten and twenty years, of the mortgage loans. The layer shown with GF has the highest risk in the figure. The other areas with two colours, green and yellow, are between other areas with blue and red colours. For example, the area CDEH shows a risk level higher than ABK but lower than GF.

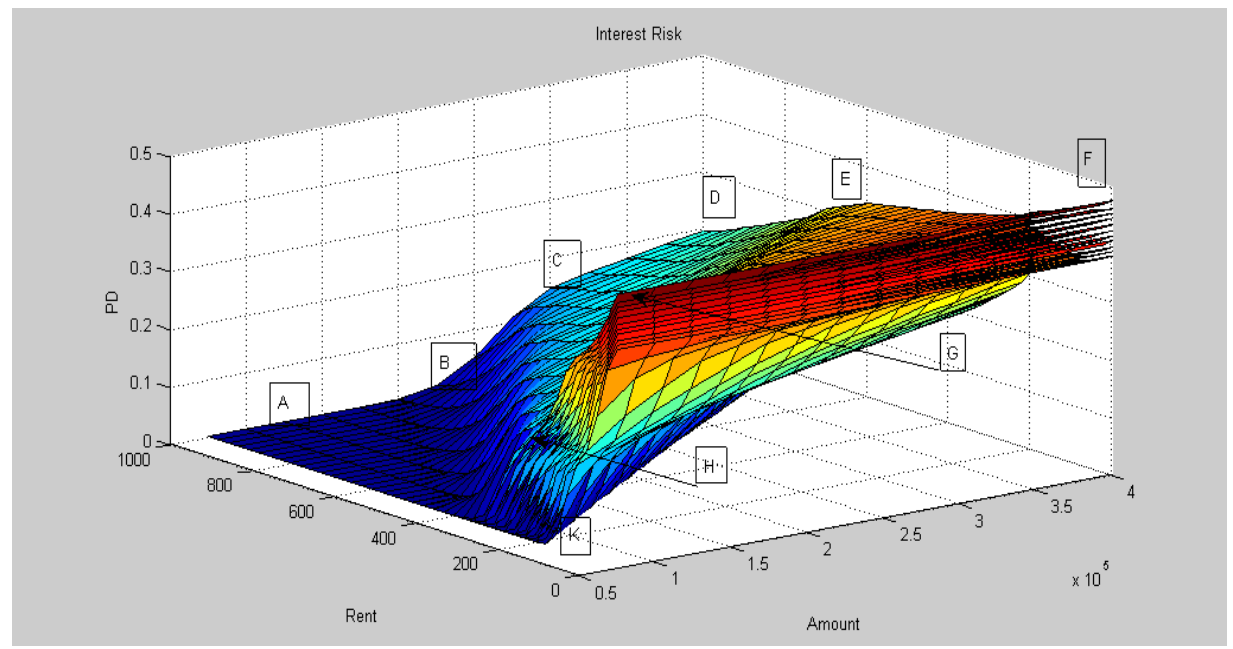

Figure 3. Probability of defaults for different levels of mortgage loans, maturites, and rent amounts

A rational investor cannot exactly determine the level of mortgage loan by only looking at Figure 3. Therefore, 
eleven tables (Table 1, Table 2, Table 3, Table 4, Table 5, Table 6, Table 7, Table 8, Table 9, Table 10, Table 11), which can be seen in the appendix, are prepared to determine the zero PD for negative net present value. For example, PD is zero for a mortgage loan amount of 75,000 T.L. and for a rent amount of 400 T.L. in Table 1. If a person thinks that the he or she will get less than 400 T.L., the PD will not be zero. In other words, the cut-off point for a mortgage loan amount of 75,000 T.L. is a 400 T.L. rent for a rational investor. For instance, if a person thinks that he or she can only get 350 rent for a house with a 75,000 T.L. mortgage loan, the PD is \%0,6. PD is shown with bold in order to show the cut-off point for the amount of mortgage and rents in each table. The other interesting point in Table 1 is that there is no zero-risk investment for a mortgage loan amount of 175,000 T.L. In other words, an investment to a house would be risky after this level of mortgage loan. This is true for the mortgage loans with the maturity of ten years. But as the maturity increases from ten years to twenty years, this amount increases to 375,000 T.L. This can be seen in Table 11. Thus, a household or a person, who is willing to make a riskless investment, can reach this goal by taking a mortgage loan with a higher maturity. The important point in here is that there is an relatively appropriate mortgage level for each amount of rent with different maturities.

\section{Conclusion}

We have developed a financial model that compares owning versus renting. It is found that buying a house by using a mortgage loan is more rational than renting. Even in the case of owner occupied housing, there is an opportunity cost for the owner of the house. Since probability of having a negative net present value is zero for most of the mortgage loan amounts, buying a house by using a mortgage loan is more rational than renting.

This study differs from other studies in terms of using a single asset in order to minimize the risk. For example, Markowitz achieved a similar return with a lower risk by forming a portfolio from the stocks of different companies. Sharpe added a risk-free asset to this portfolio and determined optimum point the efficient frontier. As it is mentioned in the literature review, other studies include house to this portfolio in order to minimize all risks related with households. No portolio is formed in order to diversify the risk for this investment, house, by using a risk-free asset. This study shows that loan can be borrowed by taking no risk (annual interest rate risk), provided that a certain level of average rent is guaranteed during the life of the loan. In other words, the return in a house would be positive if the amount of rent is more than a certain level (e.g., 400 T.L.). A rational investor can easily decide to make this investment by determining the level of rent. House as an investment tool is used to diversify the portfolio risk with other financial assets such as stocks and t-bills. A house is an important and expensive investment for most of the households in their lifetime in Turkey. This is especially true for low and middle income households. Thus, we consider the house as a single investment tool or single investment project, not as a part of a hedged portfolio.

The interest rate, the level of rent and amount of mortgage loans are all based on the economic conditions of Turkey. It is found in this study that risk of the mortgage loan can be determined by using historical simulation method based risk computation and a rational choice can be made. Thus, households can manage their risk related with buying an house by determining the level of average rent during the life of the loan.

The other finding of this study is that there is a positive correlation between the amount of mortgage loan and PD. This can be easily seen in Figure 3. There is a negative correlation between the maturity of the loan and PD. This can also be seen in the eleven tables in appendix. For instance, as can be seen in Table 1, the cut-off point of rent for a 100.000 T.L mortgage loan is 550 T.L. with the maturity of ten years. But when the maturity of the mortgage loan increases to eleven years, the cut-off point with a zero PD decreases to 500 T.L., which can be seen in Table 2. As the maturity of the loan increases to twenty years, the cut-off point for rent decreases to 275 T.L. So a household can minimize its risk by taking a small amount of mortgage with a long maturity.

There is a positive relationship between the amount of the mortgage loan and rent for a zero PD. For instance, when the amount of mortgage loan is 375.000 T.L. the cut-off point for a zero PD is 950 T.L. rent. But as the amount of the loan decreases to 75.000 T.L. the cut-off point for a zero PD becomes 200 T.L. rent. This can be seen in Table 11.

In sum, amount of mortgage loan is positively related with the rent of the house, while maturity and rent amount are inversely related. A rational investor can easily manage his or her risk by knowing this relationship among these three factors. A rational investor can easily estimate the level of risk that he or she is taking by buying a house with a mortgage loan. Besides, it would be very unrealistic to discount the cashflows with a deterministic interest rate. Thus, stochastic interest rates are used via historical simulation in this study. Since a house is an important investment for most of the households in their lifetime, this study is expected to shed some light to rational decision making in this important investment. 


\section{References}

Campbell, J. Y., \& Cocco, J. F. (2003). Household Risk Management and Optimal Mortgage Choice. Quarterly Journal of Economics, 118, 1449-1494. http://dx.doi.org/10.1162/003355303322552847

Chetty, R., \& Szeidl, A. (2010). The Effect of Housing on Portfolio Choice. NBER Working Paper, 15998, 1-48.

Cocco, J. F. (2005). Portfolio Choice in the Presence of Housing. Review of Financial Studies, 18(2), 535-567. http://dx.doi.org/10.1093/rfs/hhi006

Cocco, J. F., Gomes, F. J., \& Maenhout, P. J. (2005). Consumption and Portfolio Choice over the Life-Cycle. The Review of Financial Studies, 18(2), 491-533. http://dx.doi.org/10.1093/rfs/hhi017

Corradin, S., Fillat, J. L., \& Vergara-Alert, C. (2012). Optimal Portfolio Choice with Predictability in House Prices and Transaction Costs. European Central Bank Working Paper, 1470, 1-78. http://dx.doi.org/10.2139/ssrn.2079576

De Pooter, M. (2007). Examining the Nelson-Siegel Class of Term Structure Models: In-Sample Fit versus Out-of-Sample Forecasting Performance. SSRN Working Paper. http://dx.doi.org/10.2139/ssrn.992748

Diebold, F. X., \& Li, C. (2006). Forecasting the Term Structure of Government Bond Yields. Journal of Econometrics, 130, 337-364. http://dx.doi.org/10.1016/j.jeconom.2005.03.005

Diebold, F. X., Ji, L., \& Li, C. (2006). A Three-Factor Yield Curve Model: Non-Affine Structure, Systematic Risk Sources, and Generalized Duration. In L. Klein (Ed.), Macroeconomics, Finance and Econometrics: Essays in Memory of Albert Ando (pp. 240-274). Cheltenham, U.K. http://dx.doi.org/10.4337/9781781950500.00014

Diebold, F. X., Piazzesi, M., \& Rudebusch, G. D. (2005). Modeling Bond Yields in Finance and Macroeconomics. American Economic Review, 95, 415-420. http://dx.doi.org/10.1257/000282805774670194

Diebold, F. X., Rudebusch, S., \& Aruoba, S. (2006). The Macroeconomy and the Yield Curve. Journal of Econometrics, 131, 309-338. http://dx.doi.org/10.1016/j.jeconom.2005.01.011

Ehrmann, M., \& Ziegelmeyer, M. (2014). Household Risk Management and Actual Mortgage Choice $\mathrm{n}$ the Euro Area. European Central Bank Working Paper, 1631, 1-36.

Elton, E. J., \& Gruber, M. J. (1992). Optimal Investment Strategies with Investor Liabilities. Journal of Banking and Finance, 16, 869-890. http://dx.doi.org/10.1016/0378-4266(92)90030-4

Fabozzi, F. J., Martellini, L., \& Priaulet, P. (2005). Predictability in the Shape of the Term Structure of Interest Rates. Journal of Fixed Income, 40-53. http://dx.doi.org/10.3905/jfi.2005.523089

Fisher, J., \& Goetzmann, W. N. (2005). The Performance of Real Estate Portfolios: A Simulation Approach. Yale International Center for Finance Working Paper, 05-07, 1-24. http://dx.doi.org/10.3905/jpm.2005.593886

Fisher, S., \& Shelly, S. (2002). The Complete Idiot's Guide to Personal Finance in Your 20's and 30's (2nd ed.). Indianapolis, United States: Alpha Books.

Flavin, M., \& Yamashita, T. (1997). Owner-occupied Housing and the Composition of the Household Portfolio Over the Life Cycle. NBER Working Paper, w6389.

Flavin, M., \& Yamashita, T. (2002). Owner-Occupied Housing and the Composition of the Household Portfolio over the Life Cycle. American Economic Review, 92(1), 345-362. http://dx.doi.org/10.1257/000282802760015775

Goetzmann, W. N. (1993). The Single Family Home in the Investment Portfolio. Journal of Real Estate Finance and Economics, 6(3), 201-222. http://dx.doi.org/10.1007/BF01096958

Goetzmann, W. N., \& Ibbotson, R. (1990). The Performance of Real Estate as an Asset Class. Journal of Applied Corporate Finance, 3(1), 65-76. http://dx.doi.org/10.1111/j.1745-6622.1990.tb00196.x

Grossman, S. J., \& Laroque, G. (1990). Asset Pricing and Optimal Portfolio Choice in the Presence of Illiquid Durable Consumption Goods. Econometrica, 58(1), 25-51. http://dx.doi.org/10.2307/2938333

Heaton, J., \& Lucas, D. (2000). Portfolio Choice in the Presence of Background Risk. Economic Journal, 110, 1-26. http://dx.doi.org/10.1111/1468-0297.00488

McCulloch, J. H. (1971). Measuring the Term Structure of Interest Rates. Journal of Business, 44, 19-31. http://dx.doi.org/10.1086/295329 
McCulloch, J. H. (1975). The Tax Adjusted Yield Curve. Journal of Finance, 30, 811-830. http://dx.doi.org/10.1111/j.1540-6261.1975.tb01852.x

Molenaars, T. K., Reineirnk, N. H., \& Hemminga, M. A. (2015). Forecasting the Yield Curve - Forecast Performance of the Dynamic Nelson-Siegel Model from 1971 to 2008. Munich Personal RePEc Archive, 61862. Netherlands.

Mönch, E. (2006). Forecasting the Yield Curve in a Data-Rich Environment: A No-Arbitrage Factor-Augmented VAR Approach. Humboldt University Working paper, Berlin.

Nelson, C. R., \& Siegel, A. F. (1987). Parsimonious Modeling of Yield Curves. Journal of Business, 60, 473-489. http://dx.doi.org/10.1086/296409

Pelizzon, L., \& Weber, G. (2008). Are Household Portfolios Efficient? An Analysis Conditional on Housing. Journal of Financial and QuantitativeAnalysis, 43(2), 401-431. http://dx.doi.org/10.1017/S0022109000003574

Ross, S. A., \& Zisler, R. C. (1991). Risk and Return in Real Estate. Journal of Real Estate Finance and Economics, 4(2), 175-90. http://dx.doi.org/10.1007/bf00173123

Sinai, T., \& Souleles, N. S. (2005). Owner-Occupied Housing as a Hedge Against Rent Risk. The Quarterly Journal of Economics, 120(2), 763-789. http://dx.doi.org/10.1162/0033553053970197

Svensson, L. E. O. (1994). Estimating and Interpreting Forward Interest Rates: Sweden 1992-1994. NBER Working Paper Series, 4871. http://dx.doi.org/10.5089/9781451853759.001

Tsui, A. K. Wu, J. X., \& Zhang, Z. Y. (2013). Modelling the Term Structure of Japanese Bond Yields with the Nelson-Siegel Model. 20th International Congress on Modeling and Simulation, Adelaide, Australia, pp. 1392-1398.

Vasicek, O. A., \& Fong, H. G. (1982). Term Structure Modeling Using Exponential Splines. Journal of Finance, 37(2), 339-348. http://dx.doi.org/10.1111/j.1540-6261.1982.tb03555.x

Yao, R., \& Zhang, H. H. (2005). Optimal Consumption and Portfolio Choices with Risky Housing and Borrowing Constraints. The Review of Financial Studies, 18(1), 197-239. http://dx.doi.org/10.1093/rfs/hhh007

Zoutman, F. T. (2015). The Effect of Capital Taxation on Households' Portfolio Composition and Intertemporal Choice. CESifo Area Conference on Public Sector Economics, Germany, pp. 1-28.

\section{Appendix}

Table 1. Annual probability of defaults for different levels of mortgage loans and rents with the maturity of ten years

\begin{tabular}{|c|c|c|c|c|c|c|c|c|c|c|c|c|c|c|}
\hline & 75000 & 100000 & 125000 & 150000 & 175000 & 200000 & 225000 & 250000 & 275000 & 300000 & 325000 & 350000 & $\mathbf{3 7 5 0 0 0}$ & 400000 \\
\hline 0 & 0,476 & 0,476 & 0,476 & 0,476 & 0,476 & 0,476 & 0,476 & 0,476 & 0,476 & 0,476 & 0,476 & 0,476 & 0,476 & 0,476 \\
\hline 25 & 0,427 & 0,440 & 0,448 & 0,451 & 0,453 & 0,457 & 0,458 & 0,458 & 0,458 & 0,461 & 0,461 & 0,462 & 0,462 & 0,463 \\
\hline 50 & 0,389 & 0,409 & 0,422 & 0,427 & 0,436 & 0,440 & 0,446 & 0,448 & 0,449 & 0,451 & 0,453 & 0,453 & 0,455 & 0,457 \\
\hline 75 & 0,362 & 0,382 & 0,395 & 0,409 & 0,418 & 0,423 & 0,427 & 0,433 & 0,437 & 0,440 & 0,444 & 0,447 & 0,448 & 0,449 \\
\hline 100 & 0,344 & 0,362 & 0,379 & 0,389 & 0,398 & 0,409 & 0,416 & 0,422 & 0,425 & 0,427 & 0,433 & 0,436 & 0,438 & 0,440 \\
\hline 125 & 0,313 & 0,349 & 0,362 & 0,375 & 0,384 & 0,393 & 0,399 & 0,409 & 0,414 & 0,419 & 0,423 & 0,426 & 0,427 & 0,432 \\
\hline 150 & 0,255 & 0,333 & 0,350 & 0,362 & 0,374 & 0,382 & 0,389 & 0,395 & 0,401 & 0,409 & 0,414 & 0,418 & 0,422 & 0,423 \\
\hline 175 & 0,210 & 0,301 & 0,339 & 0,353 & 0,362 & 0,372 & 0,380 & 0,386 & 0,392 & 0,398 & 0,403 & 0,409 & 0,414 & 0,417 \\
\hline 200 & 0,183 & 0,255 & 0,320 & 0,344 & 0,355 & 0,362 & 0,370 & 0,379 & 0,383 & 0,389 & 0,394 & 0,398 & 0,403 & 0,409 \\
\hline 225 & 0,162 & 0,223 & 0,289 & 0,333 & 0,346 & 0,357 & 0,362 & 0,370 & 0,377 & 0,382 & 0,387 & 0,391 & 0,395 & 0,398 \\
\hline 250 & 0,145 & 0,194 & 0,255 & 0,313 & 0,338 & 0,349 & 0,358 & 0,362 & 0,370 & 0,375 & 0,380 & 0,384 & 0,389 & 0,393 \\
\hline 275 & 0,101 & 0,179 & 0,228 & 0,284 & 0,324 & 0,340 & 0,350 & 0,358 & 0,362 & 0,369 & 0,375 & 0,380 & 0,383 & 0,387 \\
\hline 300 & 0,050 & 0,162 & 0,203 & 0,255 & 0,307 & 0,333 & 0,344 & 0,350 & 0,358 & 0,362 & 0,368 & 0,374 & 0,379 & 0,382 \\
\hline 325 & 0,018 & 0,151 & 0,187 & 0,234 & 0,278 & 0,317 & 0,337 & 0,345 & 0,351 & 0,359 & 0,362 & 0,368 & 0,373 & 0,378 \\
\hline 350 & 0,006 & 0,129 & 0,177 & 0,210 & 0,255 & 0,301 & 0,327 & 0,339 & 0,347 & 0,353 & 0,359 & 0,362 & 0,368 & 0,372 \\
\hline 375 & 0,002 & 0,088 & 0,162 & 0,194 & 0,237 & 0,274 & 0,313 & 0,333 & 0,342 & 0,349 & 0,354 & 0,359 & 0,362 & 0,368 \\
\hline
\end{tabular}




\begin{tabular}{|c|c|c|c|c|c|c|c|c|c|c|c|c|c|c|}
\hline 400 & 000 & 050 & 154 & ,183 &, 217 & 0,255 & 0,294 & 0,320 & 0,336 & 0,344 & 0,350 & 0,355 & 0,359 & 0,362 \\
\hline 425 & 000 & 023 & & 0,174 & 0201 & & 0,272 & 0,307 & 0,328 & & 0,345 & & 0,357 & 0,359 \\
\hline 450 & 000 & 010 & & & & & & & & & & & & 0,357 \\
\hline 475 & 0,000 & 0,004 & & 0,156 & & & & & & & & & 0,348 & 0,351 \\
\hline 500 & 0,000 & 0,002 & & & & & & & & & & & 344 & \\
\hline 525 & 0,000 & 0,001 & 30 & & & & & & & & & & 39 & 0,345 \\
\hline 550 & 0,000 & 0,000 & & & & & & & & & & & & 0,340 \\
\hline 575 & 0,000 & 0,000 & & 0,076 & & & & & & & & & 330 & 0,337 \\
\hline 600 & 0,000 & 0,000 & 0,004 & 0,050 & 0,13 & & & 03 & & 255 & 0,279 & 307 & 0,320 & 0,333 \\
\hline 625 & 0,000 & 0,000 & 002 & 0,032 & 0,121 & 158 & 0,178 & 194 & & 243 & 0,267 & 294 & 313 & 0,325 \\
\hline 650 & 0,000 & 0,000 & 0,001 & 0,018 & & & & & & & & & 303 & 317 \\
\hline 675 & & 0,000 & 0,000 & & & & & & & & & & 289 & 0,310 \\
\hline 700 & 0,000 & 0,000 & 0,000 & 0,006 & 0,050 & 0.129 & 0,159 & 177 & 0,190 & 0,210 & 0,236 & 0,255 & 0,276 & 0,301 \\
\hline 725 & 0,000 & 0,000 & 0,000 & 0,003 & 0,034 & 0,109 & 0,154 & 167 & & 0,201 & 0,226 & ,246 & 0,266 & 0,287 \\
\hline 750 & 0,000 & 0,000 & 0,000 & 0,002 & 0,022 & 0,088 & 0,145 & 162 & 0,179 & 0,194 & 0,213 & 237 & 255 & 0,274 \\
\hline 775 & & & & & & & & & & & & & & \\
\hline 800 & 0,000 & 0,000 & 0,000 & & & & & & & & & & & 0,255 \\
\hline 825 & 0,000 & 0,000 & 0,000 & 0,000 & 0,005 & 0,036 & 0,101 & 0,146 & & & 0,191 & ,208 & 0,228 & 0,248 \\
\hline 850 & 0,000 & 0,000 & 0,000 & 0,000 & 0,003 & 0,023 & 0,084 & 0,140 & 0,159 & 0,174 & 0,187 & 0,201 & 0,221 & 0,238 \\
\hline 875 & 0,000 & 0,000 & & 0,000 & & & & & & & & & 0,210 & 0,230 \\
\hline 900 & 0,000 & 0,000 & & & 0,001 & & & & & & & 0,190 & 0,203 & 0,223 \\
\hline 925 & 0,000 & 0,000 & 0,000 & 0,000 & 0,000 & & & & & & & & 0,198 & 0,213 \\
\hline 950 & 0,000 & 0,000 & 0,000 & & 0,000 & & & & 0,136 & 0,156 & 0,166 & 0,180 & 0,191 & 0,206 \\
\hline 975 & 0,000 & 0,000 & 0,000 & 0,000 & 0,000 & 0,003 & & 0,063 & & & 0,162 & 0,177 & 0,187 & 0,200 \\
\hline 1000 & 0,000 & 0,000 & 0,000 & 0,000 & 0,000 & 0,002 & 0,013 & 0,050 & 0,107 & 0,145 & 0,159 & 0,171 & 0,183 & 0,194 \\
\hline
\end{tabular}

Table 2. Annual probability of defaults for different levels of mortgage loans and rents with the maturity of eleven years

\begin{tabular}{|c|c|c|c|c|c|c|c|c|c|c|c|c|c|c|}
\hline & 75000 & 00000 & 125000 & 150000 & 175000 & 200000 & 225000 & 250000 & 275000 & 300000 & 325000 & 350000 & 375000 & 400000 \\
\hline 0 & & & & & & & & & & & & & & \\
\hline 25 & & & & & & & & & & & & & & \\
\hline 50 & & & 409 & & & & & & & & & & & ,448 \\
\hline 75 & & & & & & & & & & & & & & 437 \\
\hline 100 & & & & & & & & & & & & & & 0,427 \\
\hline 125 & & & & & & & & & & & & & & 0,421 \\
\hline 150 & 0,229 & 0,314 & 0,343 & 0,357 & 0,363 & & & & & & & & & 0,413 \\
\hline 175 & & & & & & & & & & & & & & 0,402 \\
\hline 200 & 0,169 & 0,229 & 0,296 & 333 & 0,346 & & 362 & 368 & 75 & 380 & 383 & 0,387 & 391 & 0,395 \\
\hline 225 & 0,151 & & 0,257 & 314 & & & & & & & & & 384 & 0,389 \\
\hline 250 & & & & & & & & & & & & & & 0,383 \\
\hline 275 & & & & & & & & & & & & & & \\
\hline 300 & 0,022 & 0,151 & 0,187 & 0,229 & 0,272 & 0,314 & 0,333 & 343 & 350 & 357 & & & ,368 & 0,373 \\
\hline 325 & 0,007 & 128 & 0,175 & 0,207 & & & & & & & & & ,362 & 0,368 \\
\hline 350 & 0,002 & 0,085 & 0,160 & 190 & 0,229 & 0,266 & 306 & & 38 & 45 & & & 359 & 0,362 \\
\hline 375 & & & & & & & & & & & & & & 0,359 \\
\hline 400 & & & & & & & & & & & & & & 0,357 \\
\hline 425 & 0,000 & 0,009 & 0,102 & 0,160 & & & 0245 & & & & & & ,346 & 0,350 \\
\hline 450 & 0,000 & 0,004 & 0,070 & 0,151 & 0,177 & 0,199 & 0,229 & 257 & 288 & 0,314 & 0,328 & 0,337 & ,343 & 0,348 \\
\hline 475 & & & & & & & & & & & & & & 0,344 \\
\hline 500 & 0,000 & & 0,022 & & & & & 0,229 & & 0,281 & & 0,320 & 0,333 & 0,339 \\
\hline 525 & 0,000 & & 0,010 & 0,085 & & & & & & & & & 0,326 & 0,334 \\
\hline 550 & 0,000 & & 0,005 & & & & 0,183 & 0,202 & 0,229 & 0,251 & 0,276 & 0,302 & & 0,329 \\
\hline 575 & 0,000 & 0,000 & 0,002 & & 0,124 & & & & & & & & & 0,320 \\
\hline 600 & 0,000 & 0,000 & 0,001 & 0,022 & 0,097 & 0,151 & 0,169 & 0,187 & 0,205 & 0,229 & 0,250 & 0,272 & 0,296 & 0,314 \\
\hline
\end{tabular}




\begin{tabular}{|c|c|c|c|c|c|c|c|c|c|c|c|c|c|c|}
\hline 625 & 0,000 & ,000 & $\mathbf{0 , 0 0 0}$ & 0,012 & 0,076 & 0,140 & 0,161 & 0,180 & 0,197 & 0,218 & 0,239 & 0,263 & 0,281 & 0,304 \\
\hline 650 & 0,000 & 0,000 & & & & & & & & & & & & 0,290 \\
\hline 675 & 0,000 & 0,000 & 0,000 & 0,004 & 0,034 & & & & 0,183 & & & & 0,257 & 0,277 \\
\hline 700 & 0,000 & 0,000 & 0,000 & 0,002 & 0,022 & & & 160 & 0,178 & & & & 0,249 & 0,266 \\
\hline 725 & 0,000 & 0,000 & 0,000 & 0,001 & 0,013 & & & 0,157 & 0,171 & & & 0,220 & 0,238 & 0,256 \\
\hline 750 & 0,000 & 0,000 & 0,000 & $\mathbf{0 , 0 0 0}$ & 0,008 & & & 0,151 & 0,164 & 180 & 0,193 & 0,208 & 0,229 & 0,249 \\
\hline 775 & 0,000 & 0,000 & 0,000 & 0,000 & 0,004 & 0,032 & 0,095 & 0,142 & 0,160 & 0,176 & 0,187 & 0,201 & 0,221 & 0,238 \\
\hline 800 & 0,000 & 0,000 & 0,000 & 0,000 & 0,002 & 0,022 & 0,077 & 0,136 & 0,157 & 0,169 & 0,183 & 0,194 & 0,210 & 0,229 \\
\hline 825 & 0,000 & 0,000 & 0,00 & 0,000 & 0,001 & & & 122 & & 0,162 & & 0,189 & 0,202 & 0,221 \\
\hline 850 & 0,000 & & & & & & & & & & & & & 0,210 \\
\hline 875 & 0,000 & 0,000 & 0,000 & 0,000 & 0,000 & 0,006 & 0,032 & 0,085 & 0,137 & 0,156 & & & 0,190 & 0,205 \\
\hline 900 & 0,000 & 0,000 & 0,000 & 0,000 & 0,000 & 0,004 & 0,022 & 0,070 & 0,126 & 0,151 & 0,162 & 0,177 & 0,187 & 0,199 \\
\hline 925 & 0,000 & 0,000 & 0,000 & 0,000 & 0,000 & 0,002 & 0,014 & 0,053 & 0,108 & 0,145 & 0,159 & 0,171 & 0,182 & 0,193 \\
\hline 950 & 0,000 & 0,000 & 0,000 & 0,000 & 0,000 & 0,001 & 0,010 & 0,042 & 0,092 & 0,138 & & 0,164 & 0,178 & 0,187 \\
\hline 975 & 0,000 & 0,000 & 0,000 & 0,000 & 0,000 & & 0,007 & 0,031 & 0,079 & 0,128 & 0,151 & 0,160 & 0,175 & 0,183 \\
\hline 1000 & 0,000 & 0,000 & 0,000 & 0,000 & 0,000 & 0,000 & 0,004 & 0,022 & 0,062 & 0,116 & 0,145 & 0,159 & 0,169 & 0,180 \\
\hline
\end{tabular}

Table 3. Annual probability of defaults for different levels of mortgage loans and rents with the maturity of twelve years

\begin{tabular}{|c|c|c|c|c|c|c|c|c|c|c|c|c|c|c|}
\hline & 75000 & 100000 & 125000 & 150000 & 175000 & 200000 & 225000 & 250000 & 275000 & 300000 & 325000 & 350000 & 375000 & 400000 \\
\hline 0 & 0,453 & 0,453 & 0,453 & 0,453 & 0,453 & 0,453 & 0,453 & 0,453 & 0,453 & 0,453 & 0,453 & 0,453 & 0,453 & 0,453 \\
\hline 25 & 0,406 & 0,417 & 0,423 & 0,427 & 0,433 & 0,435 & 0,437 & 0,439 & 0,441 & 0,442 & 0,442 & 0,443 & 0,445 & 0,445 \\
\hline 50 & 0,370 & 0,384 & 0,395 & 0,406 & 0,413 & 0,417 & 0,422 & 0,423 & 0,425 & 0,427 & 0,429 & 0,433 & 0,434 & 0,435 \\
\hline 75 & 0,346 & 0,362 & 0,375 & 0,384 & 0,392 & 98 & 0,406 & 0,411 & & 0,417 & 0,421 & 0,422 & 0,423 & 0,425 \\
\hline 100 & 0,314 & 0,346 & 0,359 & 0,370 & 0,379 & 0,384 & 0,390 & 0,395 & 0,399 & 0,406 & 0,410 & 0,413 & 0,415 & 0,417 \\
\hline 125 & 0,250 & 0,326 & 0,346 & 0,359 & 0,364 & 0,374 & 0,380 & 0,384 & 0,390 & 0,394 & 0,397 & 0,399 & 0,406 & 0,409 \\
\hline 150 & 0,204 & 0,282 & 0,332 & 0,346 & 0,357 & 0,362 & 0,370 & 0,375 & 0,380 & 0,384 & 0,389 & 0,392 & 0,395 & 0,398 \\
\hline 175 & 0,178 & 0,239 & 0,304 & 0,334 & 0,346 & 0,356 & 0,361 & 0,367 & 0,373 & 0,378 & 0,380 & 0,384 & 0,389 & 0,391 \\
\hline 200 & 0,158 & 0,204 & 0,263 & 0,314 & 0,337 & 0,346 & 0,354 & 0,359 & 0,363 & 0,370 & 0,375 & 0,379 & 0,381 & 0,384 \\
\hline 225 & 0,129 & 0,183 & 0,233 & 0,282 & 0,320 & 0,338 & 0,346 & 0,353 & 0,359 & 0,362 & 0,368 & 0,372 & 0,375 & 0,380 \\
\hline 250 & 0,070 & 0,164 & 0,204 & 0,250 & 0,298 & 0,326 & 0,339 & 0,346 & 0,352 & 0,359 & 0,362 & 0,364 & 0,370 & 0,374 \\
\hline 275 & 0,025 & 0,152 & 0,187 & 0,228 & 0,268 & 0,307 & 0,331 & 0,340 & 0,346 & 0,351 & 0,358 & 0,361 & 0,363 & 0,368 \\
\hline 300 & 0,008 & 0,129 & 0,173 & 0,204 & 0,245 & 0,282 & 0,314 & 0,332 & 0,340 & 0,346 & 0,351 & 0,357 & 0,359 & 0,362 \\
\hline 325 & 0,002 & 0,084 & 0,160 & 0,188 & 0,223 & 0,259 & 0,294 & 0,317 & 0,333 & 0,340 & 0,346 & 0,350 & 0,357 & 0,359 \\
\hline 350 & $\mathbf{0 , 0 0 0}$ & 0,045 & 0,147 & 0,178 & 0,204 & 0,239 & 0,271 & 0,304 & 0,322 & 0,334 & 0,341 & 0,346 & 0,350 & 0,356 \\
\hline 375 & 0,000 & 0,019 & 0,129 & 0,164 & 0,190 & 0,222 & 0,250 & 0,282 & 0,310 & 0,326 & 0,336 & 0,343 & 0,346 & 0,350 \\
\hline 400 & 0,000 & 0,008 & 0,092 & 0,158 & 0,180 & 0,204 & 0,237 & 0,263 & 0,293 & 0,314 & 0,328 & 0,337 & 0,343 & 0,346 \\
\hline 425 & 0,000 & 0,002 & 0,058 & 0,145 & 0,171 & 0,190 & 0,220 & 0,248 & 0,272 & 0,301 & 0,317 & 0,332 & 0,338 & 0,343 \\
\hline 450 & 0,000 & 0,001 & 0,032 & 0,129 & 0,160 & 0,183 & 0,204 & 0,233 & 0,257 & 0,282 & 0,307 & 0,320 & 0,332 & 0,338 \\
\hline 475 & 0,000 & 0,000 & 0,015 & 0,098 & 0,154 & 0,175 & 0,193 & 0,217 & 0,242 & 0,266 & 0,291 & 0,312 & 0,323 & 0,333 \\
\hline 500 & 0,000 & 0,000 & 0,008 & 0,070 & 0,143 & 0,164 & 0,184 & 0,204 & 0,229 & 0,250 & 0,273 & 0,298 & 0,314 & 0,326 \\
\hline 525 & 0,000 & 0,000 & 0,003 & 0,045 & 0,129 & 0,159 & 0,178 & 0,193 & 0,214 & 0,239 & 0,262 & 0,282 & 0,304 & 0,316 \\
\hline 550 & 0,000 & 0,000 & 0,001 & 0,025 & 0,101 & 0,152 & 0,169 & 0,187 & 0,204 & 0,228 & 0,250 & 0,268 & 0,288 & 0,307 \\
\hline 575 & 0,000 & 0,000 & 0,000 & 0,014 & 0,079 & 0,140 & 0,161 & 0,179 & 0,194 & 0,213 & 0,237 & 0,255 & 0,274 & 0,296 \\
\hline 600 & 0,000 & 0,000 & 0,000 & 0,008 & 0,054 & 0,129 & 0,158 & 0,173 & 0,187 & 0,204 & 0,227 & 0,245 & 0,263 & 0,282 \\
\hline 625 & 0,000 & 0,000 & 0,000 & 0,004 & 0,035 & 0,106 & 0,150 & 0,164 & 0,182 & 0,194 & 0,212 & 0,236 & 0,250 & 0,269 \\
\hline 650 & 0,000 & 0,000 & 0,000 & 0,002 & 0,022 & 0,084 & 0,140 & 0,160 & 0,177 & 0,188 & 0,204 & 0,223 & 0,241 & 0,259 \\
\hline 675 & 0,000 & 0,000 & 0,000 & 0,001 & 0,013 & 0,062 & 0,129 & 0,155 & 0,168 & 0,183 & 0,197 & 0,212 & 0,233 & 0,250 \\
\hline 700 & 0,000 & 0,000 & 0,000 & 0,000 & 0,008 & 0,045 & 0,109 & 0,147 & 0,162 & 0,178 & 0,189 & 0,204 & 0,223 & 0,239 \\
\hline 725 & 0,000 & 0,000 & 0,000 & 0,000 & 0,004 & 0,030 & 0,088 & 0,140 & 0,159 & 0,171 & 0,183 & 0,197 & 0,211 & 0,230 \\
\hline 750 & 0,000 & 0,000 & 0,000 & 0,000 & 0,002 & 0,019 & 0,070 & 0,129 & 0,154 & 0,164 & 0,179 & 0,190 & 0,204 & 0,222 \\
\hline 775 & 0,000 & 0,000 & 0,000 & 0,000 & 0,001 & 0,012 & 0,052 & 0,111 & 0,146 & 0,160 & 0,175 & 0,184 & 0,197 & 0,210 \\
\hline 800 & 0,000 & 0,000 & 0,000 & 0,000 & 0,000 & 0,008 & 0,037 & 0,092 & 0,140 & 0,158 & 0,166 & 0,180 & 0,190 & 0,204 \\
\hline 825 & 0,000 & 0,000 & 0,000 & 0,000 & 0,000 & 0,005 & 0,025 & 0,077 & 0,129 & 0,152 & 0,162 & 0,177 & 0,187 & 0,198 \\
\hline
\end{tabular}




\begin{tabular}{ccccccccccccccc}
\hline $\mathbf{8 5 0}$ & 0,000 & 0,000 & 0,000 & 0,000 & 0,000 & 0,002 & 0,017 & 0,058 & 0,115 & 0,145 & 0,159 & 0,171 & 0,182 & 0,190 \\
$\mathbf{8 7 5}$ & 0,000 & 0,000 & 0,000 & 0,000 & 0,000 & 0,001 & 0,011 & 0,045 & 0,097 & 0,140 & 0,156 & 0,164 & 0,178 & 0,187 \\
$\mathbf{9 0 0}$ & 0,000 & 0,000 & 0,000 & 0,000 & 0,000 & 0,001 & 0,008 & 0,032 & 0,081 & 0,129 & 0,151 & 0,160 & 0,173 & 0,183 \\
$\mathbf{9 2 5}$ & 0,000 & 0,000 & 0,000 & 0,000 & 0,000 & $\mathbf{0 , 0 0 0}$ & 0,005 & 0,022 & 0,063 & 0,117 & 0,145 & 0,158 & 0,166 & 0,179 \\
$\mathbf{9 5 0}$ & 0,000 & 0,000 & 0,000 & 0,000 & 0,000 & 0,000 & 0,003 & 0,015 & 0,051 & 0,098 & 0,138 & 0,154 & 0,162 & 0,175 \\
$\mathbf{9 7 5}$ & 0,000 & 0,000 & 0,000 & 0,000 & 0,000 & 0,000 & 0,002 & 0,011 & 0,039 & 0,084 & 0,129 & 0,149 & 0,160 & 0,170 \\
$\mathbf{1 0 0 0}$ & 0,000 & 0,000 & 0,000 & 0,000 & 0,000 & 0,000 & $\mathbf{0 , 0 0 1}$ & $\mathbf{0 , 0 0 8}$ & $\mathbf{0 , 0 2 9}$ & $\mathbf{0 , 0 7 0}$ & $\mathbf{0 , 1 1 9}$ & $\mathbf{0 , 1 4 3}$ & $\mathbf{0 , 1 5 8}$ & $\mathbf{0 , 1 6 4}$ \\
\hline
\end{tabular}

Table 4. Annual probability of defaults for different levels of mortgage loans and rents with the maturity of thirteen years

\begin{tabular}{|c|c|c|c|c|c|c|c|c|c|c|c|c|c|c|}
\hline & 75000 & 00000 & 125000 & 150000 & 175000 & 200000 & 225000 & 250000 & 275000 & 300000 & 325000 & 350000 & 375000 & 400000 \\
\hline 0 & 0,445 & 0,445 & 0,445 & 0,445 & 0,445 & 0,445 & 0,445 & 0,445 & 0,445 & 0,445 & 0,445 & 0,445 & 0,445 & 0,445 \\
\hline 25 & & & & & & & & & & & & & & 0,434 \\
\hline 50 & 0,361 & & & & & & & & & & & & & 0,423 \\
\hline 75 & 0,338 & 0,357 & & 0,375 & & & & & & & & & & 0,415 \\
\hline 100 & 0,284 & 0,338 & 351 & 0,361 & 0,369 & 0,375 & 381 & 385 & 0,390 & 0,393 & 0,395 & 0,399 & 402 & 0,406 \\
\hline 125 & 0,228 & 0,304 & 338 & 0,350 & 0,359 & 0,363 & 370 & 0,375 & 0,380 & 0,383 & 0,388 & 0,390 & 393 & 0,395 \\
\hline 150 & 0,187 & 0,251 & 0,313 & 0,338 & 0,348 & 0,357 & 0,361 & 366 & 0,372 & 0,375 & 0,380 & 0,383 & 385 & 0,390 \\
\hline 175 & 0,162 & 0,212 & 0,270 & 0,316 & 0,338 & 0,346 & 0,354 & 0,359 & 0,362 & 0,368 & 0,373 & 0,375 & 379 & 0,382 \\
\hline 200 & & & & & & 0,338 & & & 358 & 0,361 & & & & 375 \\
\hline 225 & 0,084 & 0,1 & & & & & & & & & & 362 & 366 & 0,370 \\
\hline 250 & 0,032 & 0,154 & 187 & 0,228 & 0,265 & 0,304 & 0,325 & 338 & 345 & 350 & 0,354 & 0,359 & 0,361 & 0,363 \\
\hline 275 & 0,009 & 0,132 & 0,171 & & & & & ,327 & & & & 0,353 & 358 & 0,359 \\
\hline 300 & 0,002 & 0,084 & 0,159 & 0,187 & 0,219 & 0,251 & 0,284 & 0,313 & 0,328 & 0,338 & 0,344 & 0,348 & 0,351 & 0,357 \\
\hline 325 & 0,000 & 0,044 & & & & & & & & & & & & 0,350 \\
\hline 350 & 0,000 & 0,018 & & 0,162 & & & & & & & & & & 0,346 \\
\hline 375 & 0,000 & 0,007 & 0,084 & & & & & & & & & & & 0,343 \\
\hline 400 & 0,000 & 0,002 & 0,050 & 0,140 & & & & 238 & & & & & 332 & 0,338 \\
\hline 425 & 0,000 & $\mathbf{0 , 0 0 0}$ & 0,023 & & & & & & & & & & & 0,332 \\
\hline 450 & 0,000 & $0,0 \mathrm{C}$ & & & & & & & & & & & 313 & \\
\hline 475 & 0,000 & 0,000 & 0,006 & 0,054 & 0,136 & 0,160 & 0,178 & ,194 & 215 & 0,239 & 0,259 & 0,278 & ,301 & 0,314 \\
\hline 500 & 0,000 & 0,000 & 0,002 & 0,032 & 0,111 & 0,154 & ,171 & ,187 & 203 & 0,228 & 0,246 & 0,265 & 284 & 0,304 \\
\hline 525 & 0,000 & 0,000 & 0,001 & 0,018 & 0,084 & 0,144 & ,162 & 179 & 0,193 & & & & 270 & 0,288 \\
\hline 550 & 0,000 & 0,000 & $\mathbf{0 , 0 0 0}$ & 0,009 & 0,057 & 0,132 & 0,158 & 171 & 0,187 & & & 0,239 & 258 & 0,274 \\
\hline 575 & 0,000 & 0,000 & 0,000 & 0,005 & 0,036 & 0,1 & 49 & 164 & 0,179 & 92 & 0,209 & 0,229 & 247 & 0,263 \\
\hline 600 & 0,000 & 0,000 & 0,000 & 0,002 & 0,022 & 0,0 & 140 & 159 & 74 & & 200 & 219 & 238 & 0,251 \\
\hline 625 & 0,000 & 0,000 & 0,000 & 0,001 & 0,013 & 0,061 & & 154 & 65 & & & ,207 & 228 & 0,241 \\
\hline 650 & 0,000 & 0,000 & & & & & & & & & & 199 & 215 & 233 \\
\hline 675 & 0,000 & & & & & & & & & & & & & \\
\hline 700 & & 0,000 & & & & & & & & & & & & 0,212 \\
\hline 725 & & & & & & & & & & & & & & \\
\hline 750 & 0,000 & 0,000 & & & & & & & & & & & & \\
\hline 775 & 0,000 & 0,000 & 0,000 & & & & & & & & & & & 0,190 \\
\hline 800 & 0,000 & 0,000 & 0,000 & 0,000 & 0,000 & 0,002 & 0,014 & 0,050 & 0,100 & 0,140 & 0,157 & 0,164 & 0,177 & 0,187 \\
\hline 825 & 0,000 & 0,000 & 0,000 & 0,000 & 0,000 & 0,001 & 0,009 & 0,036 & 0,084 & 0,132 & 0,151 & 0,160 & 0,171 & 0,182 \\
\hline 850 & 0,000 & 0,000 & 0,000 & 0,000 & 0,000 & $\mathbf{0 , 0 0 0}$ & 0,006 & 0,023 & 0,068 & 0,119 & 0,145 & 0,158 & 0,166 & 0,178 \\
\hline 875 & 0,000 & 0,000 & 0,000 & 0,000 & 0,000 & 0,000 & 0,004 & 0,018 & 0,052 & 0,099 & 0,138 & 0,154 & 0,162 & 0,173 \\
\hline 900 & 0,000 & 0,000 & 0,000 & 0,000 & 0,000 & 0,000 & & 0,011 & 0,040 & 0,084 & 0,129 & 0,146 &, 159 & 0,167 \\
\hline 925 & 0,000 & 0,000 & 0,000 & 0,000 & 0,000 & 0,000 & 0,001 & 0,009 & 0,029 & 0,069 & 0,116 & 0,140 & 0,157 & 0,162 \\
\hline 950 & 0,000 & 0,000 & 0,000 & 0,000 & 0,000 & 0,000 & 0,001 & 0,006 & 0,021 & 0,054 & 0,098 & 0,136 & 0,152 & 0,160 \\
\hline 975 & 0,000 & 0,000 & 0,000 & 0,000 & 0,000 & 0,000 & $\mathbf{0 , 0 0 0}$ & 0,003 & 0,015 & 0,044 & 0,084 & 0,126 & 0,145 & 0,158 \\
\hline 1000 & 0,000 & 0,000 & 0,000 & 0,000 & 0,000 & 0,000 & 0,000 & 0,002 & 0,010 & 0,032 & 0,070 & 0,111 & 0,140 & 0,154 \\
\hline
\end{tabular}


Table 5. Annual probability of defaults for different levels of mortgage loans and rents with the maturity of fourteen years

\begin{tabular}{|c|c|c|c|c|c|c|c|c|c|c|c|c|c|c|}
\hline & 5000 & 00000 & 125000 & 150000 & 175000 & 200000 & 225000 & 250000 & 275000 & 300000 & 325000 & 350000 & 375000 & 400000 \\
\hline 0 & & 0,434 & 0,434 & 0,434 & & 0,434 & 0,434 & & & & & & & 0,434 \\
\hline 25 & 0,383 & & & & & & & & & & & & & 0,422 \\
\hline 50 & & & 0,377 & & & & & & & & & & & 0,414 \\
\hline 75 & & & & & & & & & & & & & & 0,403 \\
\hline 100 & 0,252 & 0,320 & 0,344 & 0,353 & 0,361 & 0,367 & 0,373 & 0,377 & 0,380 & 0,383 & 0,387 & 0,390 & 0,391 & 0,393 \\
\hline 125 & 0,200 & 0,269 & 0,320 & 0,340 & 0,350 & 0,357 & 0,362 & 0,367 & & & 0,379 & 0,381 &, 383 & 0,386 \\
\hline 150 & 0,171 & 0,227 & 0,278 & 0,320 & 0,338 & 0,347 & 0,353 & 0,359 & 0,362 & 0,367 & 0,370 & 0,374 & 0,377 & 0,379 \\
\hline 175 & 0,148 & 0,190 & 0,240 & 0,288 & 0,320 & 0,337 & 0,345 & 0,350 & 0,357 & 0,359 &, 362 & 0,367 & 0,370 & 0,373 \\
\hline 200 & 0,101 & 0,171 & 0,208 & 0,252 & 0,294 & 0,320 & 0,336 & 0,344 & 0,349 & 0,353 & 0,359 & 0,361 & 0,362 & 0,367 \\
\hline 225 & 0,042 & & & & & & & & 0,343 & &, 350 & ,357 & ,359 & 0,361 \\
\hline 250 & 0,011 & 0,134 & & & & 0,269 & 02 & 320 & & 0,340 & 345 & 0,350 & 0,353 & 0,357 \\
\hline 275 & 0,002 & 0,084 & 0,158 & 0,183 & 0,213 & 0,246 & 0,274 & 0,306 & 320 & 0,333 & 340 & 0,345 & 0,349 & 0,351 \\
\hline 300 & $\mathbf{0 , 0 0 0}$ & 0,042 & 0,143 & 0,171 & 0,194 & 0,227 & 0,252 & 0,278 & 0,307 & 0,320 & 0,333 & 0,338 & 0,344 & 0,347 \\
\hline 325 & 0,000 & 0,016 & 0,119 & 0,160 & 0,182 & 0,206 & 0,236 & 0,261 & 0,284 & 0,307 & 0,320 & 0,332 & 0,338 & 0,343 \\
\hline 350 & 0,000 & 0,006 & 0,077 & 0,148 & 0,171 & 0,190 & & 0,240 & & 0,288 & 309 & 0,320 & 0,332 & 0,337 \\
\hline 375 & 0,000 & 0,001 & 0,042 & 0,134 & 0,160 & 0,182 & 0,200 & 0,227 & & 0,269 & 0,293 & 0,311 & 0,320 & 0,332 \\
\hline 400 & 0,000 & 0,000 & 0,020 & 0,101 & 0,153 & 0,171 & 0,189 & 0,208 & & 0,252 & & 0,294 &, 312 & 0,320 \\
\hline 425 & 0,000 & 0,000 & & & & & & & & & & & 297 & 0,312 \\
\hline 450 & 0,000 & 0,000 & & & & & & & & & & & & 0,298 \\
\hline 475 & 0,000 & 0,000 & & & & & & & & & & & & \\
\hline 500 & 0,000 & 0,000 & 0,000 & 0,011 & 0,065 & 0,134 & 0,158 & 0,171 & 0,185 & 0,200 & 0,219 & 0,238 & 0,252 & 0,269 \\
\hline 525 & 0,000 & 0,000 & 0,000 & 0,006 & 0,042 & 0,111 & 0,148 & 0,163 & 0,179 & 0,190 & 0,207 & 0,227 & 0,240 & 0,257 \\
\hline 550 & 0,000 & 0,000 & 0,000 & 0,002 & 0,023 & 0,084 & 0,140 & 0,158 & 0,171 & 0,183 & 0,198 & 0,213 & 0,230 & 0,246 \\
\hline 575 & 0,000 & 0,000 & 0,000 & 0,001 & 0,013 & 0,060 & 0,125 & 0,152 & 0,163 & 0,178 & 0,189 & 0,202 & 0,221 & 0,237 \\
\hline 600 & 0,000 & 0,000 & 0,000 & 0,000 & 0,008 & 0,042 & 0,101 & 0,143 & 0,159 & 0,171 & 0,183 & 0,194 & 0,208 & 0,227 \\
\hline 625 & 0,000 & 0,000 & 0,000 & 0,000 & 0,004 & 0,024 & 0,081 & 0,134 & 0,154 & 0,164 & 0,178 & 0,188 & 0,200 & 0,213 \\
\hline 650 & 0,000 & 0,000 & 0,000 & 0,000 & 0,002 & 0,016 & 0,057 & 0,119 & 0,146 & 0,160 & 0,171 & 0,182 & 0,191 & 0,206 \\
\hline 675 & 0,000 & 0,000 & 0,000 & 0,000 & 0,001 & 0,010 & 0,042 & 0,095 & & 0,157 & 164 & 177 & 0,187 & 0,198 \\
\hline 700 & & & & & & & & & & & & & & 190 \\
\hline 725 & 0,000 & & & & & & & & & & & & 0,177 & 0,186 \\
\hline 750 & 0,000 & & & & & & & & & & & & & 0,182 \\
\hline 775 & 0,000 & 0,000 & 0,000 & & 0,000 & 0,001 & 0,008 & & & & & & 0,165 & 0,177 \\
\hline 800 & 0,000 & 0,000 & & & 0,000 & $\mathbf{0 , 0 0 0}$ & & & & 0,101 & & 0,153 & 0,161 & 0,171 \\
\hline 825 & 0,000 & & & & & 0,000 & & & & 0,084 & & & & 0,166 \\
\hline 850 & 0,000 & 0,000 & 0,000 & 0,000 & 0,000 & 0,000 & 0,001 & 0,009 & 0,030 & 0,069 & 0,114 & 0,140 & 0,155 & 0,162 \\
\hline 875 & 0,000 & 0,000 & 0,000 & 0,000 & 0,000 & 0,000 & 0,001 & 0,006 & 0,022 & 0,053 & 0,097 & 0,134 & 0,148 & 0,159 \\
\hline 900 & 0,000 & 0,000 & 0,000 & 0,000 & 0,000 & 0,000 & $\mathbf{0 , 0 0 0}$ & 0,003 & 0,014 & 0,042 & 0,083 & 0,123 & 0,143 & 0,157 \\
\hline 925 & 0,000 & 0,000 & 0,000 & 0,000 & 0,000 & 0,000 & 0,000 & 0,002 & 0,010 & 0,031 & 0,068 & 0,105 & 0,138 & 0,152 \\
\hline 950 & 0,000 & 0,000 & 0,000 & 0,000 & 0,000 & 0,000 & 0,000 & 0,001 & 0,008 & 0,022 & 0,053 & 0,091 & 0,129 & 0,145 \\
\hline 975 & 0,000 & 0,000 & 0,000 & & 0,000 & 0,000 & 0,000 & 0,001 & 0,005 & 0,016 & 0,042 & 0,079 & 0,119 & 0,140 \\
\hline 1000 & 0,000 & 0,000 & 0,000 & 0,000 & 0,000 & 0,000 & 0,000 & $\mathbf{0 , 0 0 0}$ & 0,002 & 0,011 & 0,032 & 0,065 & 0,101 & $\mathbf{0 , 1 3 4}$ \\
\hline
\end{tabular}


Table 6. Annual probability of defaults for different levels of mortgage loans and rents with the maturity of fifteen years

\begin{tabular}{|c|c|c|c|c|c|c|c|c|c|c|c|c|c|c|}
\hline & 5000 & 00000 & 125000 & 150000 & 175000 & 200000 & 225000 & 250000 & 275000 & 300000 & 325000 & 350000 & 375000 & 400000 \\
\hline 0 & 0,414 & & 0,414 & & & & & & & & & & & \\
\hline 25 & 0,366 & & & & & & & & & & 0,398 &, 400 & ,402 & 0,402 \\
\hline 50 & 0,337 & & & & & & & & & & & 0,391 & ,391 & 0,391 \\
\hline 75 & & & & & & & & & & & & & & 0,383 \\
\hline 100 & 0,204 & 0,268 & 0,316 & 0,337 & 0,345 & 0,350 & 0,357 & 0,359 & 0,362 & 0,366 & 0,369 & 0,373 &, 374 & 0,376 \\
\hline 125 & 0,172 & 0,219 & 0,268 & 0,312 & 0,332 & 0,340 & & 0,350 & 0,357 & 0,359 & 0,361 & 0,363 & ,366 & 0,368 \\
\hline 150 & 0,145 & 0,186 & 0,229 & 0,268 & 0,307 & 0,325 & 0,337 & 0,344 & 0,348 & 0,350 & 0,356 & 0,359 & 0,359 & 0,362 \\
\hline 175 & 0,085 & 0,163 & 0,194 & 0,236 & 0,268 & 0,302 & 0,320 & 0,333 & 0,340 & 0,345 & 0,348 & 0,350 &, 355 & 0,359 \\
\hline 200 & 0,026 & 0,145 & 0,178 & 0,204 & 0,238 & 0,268 & 0,298 & 0,316 & 0,331 & 0,337 & 0,342 & 0,345 & 0,349 & 0,350 \\
\hline 225 & 0,0 & & & 0,186 & 0,21 & 0,240 & 0,268 & & 0,314 & 0,325 & 0,333 & 0,339 & ,344 & 0,346 \\
\hline 250 & 0,001 & 0,052 & 145 & 0,172 & & 0,219 & & 268 & & 0,312 & 0,320 & 0,332 & 0,337 & 0,340 \\
\hline 275 & 0,000 & 0,020 & 0,120 & 0,159 & 0,180 & 0,199 & 0,225 & 0,248 & 0,268 & 0,289 & 0,307 & 0,317 & 0,329 & 0,334 \\
\hline 300 & 0,000 & 0,006 & 0,074 & 0,145 & 0,166 & 0,186 & 0,204 & 0,229 & 0,250 & 0,268 & 0,287 & 0,307 & 0,316 & 0,325 \\
\hline 325 & 0,000 & 0,001 & 0,034 & 0,125 & 0,158 & 0,177 & 0,191 & 0,209 & 0,232 & 0,250 & 0,268 & 0,285 & 0,304 & 0,314 \\
\hline 350 & 0,000 & 0,000 & 0,015 & 0,085 & 0,145 & 0,163 & 0,181 & 0,194 & 0,213 & 0,236 & 0,250 & 0,268 & 0,284 & 0,302 \\
\hline 375 & 0,000 & 0,000 & 0,006 & 0,052 & 0,130 & 0,158 & 0,172 & 0,186 & 0,200 & 0,219 & 0,238 & 0,251 & 0,268 & 0,284 \\
\hline 400 & 0,000 & 0,000 & 0,001 & 0,026 & 0,098 & 0,145 & 0,162 & 0,178 & 0,190 & 0,204 & 0,223 & & ,252 & 0,268 \\
\hline 425 & 0,000 & 0,000 & 0,000 & & & & & & & & & & 239 & 0,254 \\
\hline 450 & & 0,000 & & & & & & & & & & & & 0,240 \\
\hline 475 & 0,000 & 0,000 & & & 0,022 & & & & & & & & 214 & 0,230 \\
\hline 500 & 0,000 & 0,000 & 0,000 & 0,001 & 0,011 & 0,052 & 0,114 & 0,145 & 0,159 & 0,172 & 0,183 & 0,191 & 0,204 & 0,219 \\
\hline 525 & 0,000 & 0,000 & 0,000 & 0,000 & 0,006 & 0,032 & 0,085 & 0,135 & 0,153 & 0,163 & 0,177 & 0,186 & 0,194 & 0,207 \\
\hline 550 & 0,000 & 0,000 & 0,000 & 0,000 & 0,002 & 0,020 & 0,064 & 0,120 & 0,145 & 0,159 & 0,168 & 0,180 & 0,189 & 0,199 \\
\hline 575 & 0,000 & 0,000 & 0,000 & 0,000 & 0,001 & 0,011 & 0,043 & 0,093 & 0,138 & 0,152 & 0,162 & 0,174 & 0,183 & 0,191 \\
\hline 600 & 0,000 & 0,000 & 0,000 & 0,000 & 0,000 & 0,006 & 0,026 & 0,074 & 0,122 & 0,145 & 0,158 & 0,166 & 0,178 & 0,186 \\
\hline 625 & 0,000 & 0,000 & 0,000 & 0,000 & 0,000 & 0,002 & 0,018 & 0,052 & 0,100 & 0,138 & 0,152 & 0,161 & 0,172 & 0,180 \\
\hline 650 & 0,000 & 0,000 & 0,000 & 0,000 & 0,000 & 0,001 & 0,011 & 0,034 & 0,082 & 0,125 & 0,145 & 0,158 & 0,164 & 0,177 \\
\hline 675 & 0,000 & 0,000 & 0,000 & 0,000 & 0,000 & 0,000 & ,006 & 022 & 60 & 0,104 & 0,139 & 152 & 160 & 0,168 \\
\hline 700 & & & & & & & & & & & & & & 163 \\
\hline 725 & 0,000 & & & & & & & & & & & & & 0,159 \\
\hline 750 & & & & & & & & & & & & & & 0,158 \\
\hline 775 & 0,000 & 0,000 & 0,000 & 0,000 & & 0,000 & $\mathbf{0 , 0 0 0}$ & & & & & & 0,140 & 0,152 \\
\hline 800 & 0,000 & 0,000 & 0,000 & 0,000 & & 0,000 & & & & 0,026 & & & 0,131 & 0,145 \\
\hline 825 & 0,000 & & & 0,000 & & 0,000 & 0,000 & & & & & & & 0,140 \\
\hline 850 & 0,000 & 0,000 & 0,000 & 0,000 & 0,000 & 0,000 & 0,000 & $\mathbf{0 , 0 0 0}$ & 0,003 & 0,013 & 0,033 & 0,068 & 0,100 & 0,133 \\
\hline 875 & 0,000 & 0,000 & 0,000 & 0,000 & 0,000 & 0,000 & 0,000 & 0,000 & 0,002 & 0,010 & 0,023 & 0,052 & 0,085 & 0,122 \\
\hline 900 & 0,000 & 0,000 & 0,000 & 0,000 & 0,000 & 0,000 & 0,000 & 0,000 & 0,001 & 0,006 & 0,018 & 0,041 & 0,074 & 0,104 \\
\hline 925 & 0,000 & 0,000 & 0,000 & 0,000 & 0,000 & 0,000 & 0,000 & 0,000 & $\mathbf{0 , 0 0 0}$ & 0,003 & 0,012 & 0,031 & 0,058 & 0,088 \\
\hline 950 & 0,000 & 0,000 & 0,000 & 0,000 & 0,000 & 0,000 & 0,000 & 0,000 & 0,000 & 0,002 & 0,010 & 0,022 & 0,046 & 0,079 \\
\hline 975 & 0,000 & 0,000 & 0,000 & 0,000 & 0,000 & 0,000 & & 0,000 & 0,000 & 0,001 & 0,006 & 0,017 & 0,034 & 0,066 \\
\hline 1000 & 0,000 & 0,000 & 0,000 & 0,000 & 0,000 & 0,000 & 0,000 & 0,000 & 0,000 & 0,001 & $\mathbf{0 , 0 0 3}$ & 0,011 & 0,026 & 0,052 \\
\hline
\end{tabular}

Table 7. Annual probability of defaults for different levels of mortgage loans and rents with the maturity of sixteen years

\begin{tabular}{|c|c|c|c|c|c|c|c|c|c|c|c|c|c|c|}
\hline & 75000 & 100000 & 125000 & 150000 & 175000 & 200000 & 225000 & 250000 & 275000 & 300000 & 325000 & 350000 & 375000 & 400000 \\
\hline 0 & 0,423 & 0,423 & 0,423 & 0,423 & 0,423 & 0,423 & 0,423 & 0,423 & 0,423 & 0,423 & 0,423 & 0,423 & 0,423 & 0,423 \\
\hline 25 & 0,375 & 0,383 & 0,391 & 0,395 & 0,397 & 0,401 & 0,406 & 0,408 & 0,410 & 0,410 & 0,411 & 0,412 & 0,413 & 0,413 \\
\hline 50 & 0,345 & 0,359 & 0,368 & 0,375 & 0,380 & 0,383 & 0,389 & 0,391 & 0,392 & 0,395 & 0,396 & 0,397 & 0,399 & 0,401 \\
\hline 75 & 0,295 & 0,337 & 0,350 & 0,359 & 0,363 & 0,370 & 0,375 & 0,379 & 0,382 & 0,383 & 0,387 & 0,390 & 0,391 & 0,391 \\
\hline
\end{tabular}




\begin{tabular}{|c|c|c|c|c|c|c|c|c|c|c|c|c|c|c|}
\hline 100 & 0,226 & 0,295 & 0,332 & 0,345 & 0,352 & 0,359 & 0,362 & 0,368 & 0,371 & 0,375 & 0,378 & 0,380 & 0,382 & 0,383 \\
\hline 125 & 0,183 & 0,239 & 0,295 & 0,328 & 0,340 & 0,348 & 0,354 & 0,359 & 0,362 & 0,364 & 0,368 & 0,372 & 0,375 & 0,377 \\
\hline 150 & 0,158 & 0,199 & 0,250 & 0,295 & 0,323 & 0,337 & 0,345 & 0,350 & 0,355 & 0,359 & 0,361 & 0,363 & 0,368 & 0,370 \\
\hline 175 & 0,125 & 0,177 & 0,213 & 0,254 & 0,295 & 0,319 & 0,334 & 0,342 & 0,347 & 0,350 & 0,357 & 0,359 & 0,361 & 0,362 \\
\hline 200 & 0,054 & 0,158 & 0,189 & 0,226 & 0,262 & 0,295 & 0,317 & 0,332 & 0,340 & 0,345 & 0,349 & 0,352 & 0,357 & 0,359 \\
\hline 225 & 0,014 & 0,137 & 0,171 & 0,199 & 0,235 & 0,264 & 0,295 & 0,315 & 0,331 & 0,337 & 0,343 & 0,346 & 0,350 & 0,353 \\
\hline 250 & 0,002 & 0,086 & 0,158 & 0,183 & 0,208 & 0,239 & 0,266 & 0,295 & 0,314 & 0,328 & 0,335 & 0,340 & 0,345 & 0,348 \\
\hline 275 & 0,000 & 0,042 & 0,140 & 0,168 & 0,191 & 0,218 & 0,246 & 0,269 & 0,295 & 0,314 & 0,325 & 0,333 & 0,338 & 0,344 \\
\hline 300 & 0,000 & 0,014 & 0,108 & 0,158 & 0,179 & 0,199 & 0,226 & 0,250 & 0,271 & 0,295 & 0,313 & 0,323 & 0,332 & 0,337 \\
\hline 325 & 0,000 & 0,005 & 0,068 & 0,145 & 0,166 & 0,186 & 0,206 & 0,231 & 0,251 & 0,272 & 0,295 & 0,312 & 0,320 & 0,332 \\
\hline 350 & 0,000 & 0,001 & 0,033 & 0,125 & 0,158 & 0,177 & 0,191 & 0,213 & 0,237 & 0,254 & 0,273 & 0,295 & 0,312 & 0,319 \\
\hline 375 & 0,000 & $\mathbf{0 , 0 0 0}$ & 0,014 & 0,086 & 0,146 & 0,164 & 0,183 & 0,199 & 0,221 & 0,239 & 0,258 & 0,274 & 0,295 & 0,309 \\
\hline 400 & 0,000 & 0,000 & 0,006 & 0,054 & 0,132 & 0,158 & 0,174 & 0,189 & 0,205 & 0,226 & 0,242 & 0,262 & 0,276 & 0,295 \\
\hline 425 & 0,000 & 0,000 & 0,002 & 0,029 & 0,101 & 0,147 & 0,163 & 0,180 & 0,192 & 0,210 & 0,229 & 0,246 & 0,263 & 0,277 \\
\hline 450 & 0,000 & 0,000 & 0,000 & 0,014 & 0,073 & 0,137 & 0,158 & 0,171 & 0,185 & 0,199 & 0,215 & 0,235 & 0,250 & 0,264 \\
\hline 475 & 0,000 & 0,000 & 0,000 & 0,008 & 0,046 & 0,116 & 0,149 & 0,163 & 0,178 & 0,190 & 0,204 & 0,223 & 0,238 & 0,250 \\
\hline 500 & 0,000 & 0,000 & 0,000 & 0,002 & 0,025 & 0,086 & 0,140 & 0,158 & 0,170 & 0,183 & 0,194 & 0,208 & 0,226 & 0,239 \\
\hline 525 & 0,000 & 0,000 & 0,000 & 0,001 & 0,014 & 0,060 & 0,125 & 0,151 & 0,162 & 0,177 & 0,188 & 0,199 & 0,213 & 0,229 \\
\hline 550 & 0,000 & 0,000 & 0,000 & 0,000 & 0,009 & 0,042 & 0,098 & 0,140 & 0,158 & 0,168 & 0,181 & 0,191 & 0,202 & 0,218 \\
\hline 575 & 0,000 & 0,000 & 0,000 & 0,000 & 0,003 & 0,023 & 0,077 & 0,130 & 0,152 & 0,162 & 0,175 & 0,184 & 0,194 & 0,207 \\
\hline 600 & 0,000 & 0,000 & 0,000 & 0,000 & 0,001 & 0,014 & 0,054 & 0,108 & 0,143 & 0,158 & 0,167 & 0,179 & 0,189 & 0,199 \\
\hline 625 & 0,000 & 0,000 & 0,000 & 0,000 & 0,001 & 0,009 & 0,034 & 0,086 & 0,134 & 0,152 & 0,162 & 0,173 & 0,183 & 0,191 \\
\hline 650 & 0,000 & 0,000 & 0,000 & 0,000 & 0,000 & 0,005 & 0,022 & 0,068 & 0,120 & 0,145 & 0,158 & 0,166 & 0,178 & 0,186 \\
\hline 675 & 0,000 & 0,000 & 0,000 & 0,000 & 0,000 & 0,002 & 0,014 & 0,048 & 0,097 & 0,137 & 0,152 & 0,161 & 0,171 & 0,182 \\
\hline 700 & 0,000 & 0,000 & 0,000 & 0,000 & 0,000 & 0,001 & 0,010 & 0,033 & 0,079 & 0,125 & 0,145 & 0,158 & 0,164 & 0,177 \\
\hline 725 & 0,000 & 0,000 & 0,000 & 0,000 & 0,000 & $\mathbf{0 , 0 0 0}$ & 0,006 & 0,022 & 0,058 & 0,104 & 0,139 & 0,152 & 0,160 & 0,171 \\
\hline 750 & 0,000 & 0,000 & 0,000 & 0,000 & 0,000 & 0,000 & 0,002 & 0,014 & 0,045 & 0,086 & 0,129 & 0,146 & 0,158 & 0,164 \\
\hline 775 & 0,000 & 0,000 & 0,000 & 0,000 & 0,000 & 0,000 & 0,001 & 0,010 & 0,031 & 0,071 & 0,113 & 0,140 & 0,152 & 0,160 \\
\hline 800 & 0,000 & 0,000 & 0,000 & 0,000 & 0,000 & 0,000 & 0,001 & 0,006 & 0,022 & 0,054 & 0,094 & 0,132 & 0,146 & 0,158 \\
\hline 825 & 0,000 & 0,000 & 0,000 & 0,000 & 0,000 & 0,000 & 0,000 & 0,003 & 0,014 & 0,042 & 0,080 & 0,120 & 0,140 & 0,153 \\
\hline 850 & 0,000 & 0,000 & 0,000 & 0,000 & 0,000 & 0,000 & 0,000 & 0,002 & 0,010 & 0,029 & 0,064 & 0,101 & 0,134 & 0,147 \\
\hline 875 & 0,000 & 0,000 & 0,000 & 0,000 & 0,000 & 0,000 & 0,000 & 0,001 & 0,006 & 0,021 & 0,050 & 0,086 & 0,125 & 0,142 \\
\hline 900 & 0,000 & 0,000 & 0,000 & 0,000 & 0,000 & 0,000 & 0,000 & $\mathbf{0 , 0 0 0}$ & 0,004 & 0,014 & 0,036 & 0,073 & 0,108 & 0,137 \\
\hline 925 & 0,000 & 0,000 & 0,000 & 0,000 & 0,000 & 0,000 & 0,000 & 0,000 & 0,002 & 0,010 & 0,026 & 0,057 & 0,093 & 0,128 \\
\hline 950 & 0,000 & 0,000 & 0,000 & 0,000 & 0,000 & 0,000 & 0,000 & 0,000 & 0,001 & 0,008 & 0,021 & 0,046 & 0,081 & 0,116 \\
\hline 975 & 0,000 & 0,000 & 0,000 & 0,000 & 0,000 & 0,000 & 0,000 & 0,000 & 0,001 & 0,005 & 0,014 & 0,033 & 0,068 & 0,099 \\
\hline 1000 & 0,000 & 0,000 & 0,000 & 0,000 & 0,000 & 0,000 & 0,000 & 0,000 & $\mathbf{0 , 0 0 0}$ & $\mathbf{0 , 0 0 2}$ & 0,011 & $\mathbf{0 , 0 2 5}$ & 0,054 & 0,086 \\
\hline
\end{tabular}

Table 8. Annual probability of defaults for different levels of mortgage loans and rents with the maturity of seventeen years

\begin{tabular}{cccccccccccccccc}
\hline & $\mathbf{7 5 0 0 0}$ & $\mathbf{1 0 0 0 0 0}$ & $\mathbf{1 2 5 0 0 0}$ & $\mathbf{1 5 0 0 0 0}$ & $\mathbf{1 7 5 0 0 0}$ & $\mathbf{2 0 0 0 0 0}$ & $\mathbf{2 2 5 0 0 0}$ & $\mathbf{2 5 0 0 0 0}$ & $\mathbf{2 7 5 0 0 0}$ & $\mathbf{3 0 0 0 0 0}$ & $\mathbf{3 2 5 0 0 0}$ & $\mathbf{3 5 0 0 0 0}$ & $\mathbf{3 7 5 0 0 0}$ & $\mathbf{4 0 0 0 0 0}$ \\
\hline $\mathbf{0}$ & 0,406 & 0,406 & 0,406 & 0,406 & 0,406 & 0,406 & 0,406 & 0,406 & 0,406 & 0,406 & 0,406 & 0,406 & 0,406 & 0,406 & 0,406 \\
$\mathbf{2 5}$ & 0,359 & 0,368 & 0,375 & 0,379 & 0,382 & 0,383 & 0,386 & 0,389 & 0,390 & 0,391 & 0,391 & 0,391 & 0,391 & 0,392 \\
$\mathbf{5 0}$ & 0,324 & 0,345 & 0,354 & 0,359 & 0,363 & 0,368 & 0,372 & 0,375 & 0,377 & 0,379 & 0,380 & 0,382 & 0,382 & 0,383 & 0,375 \\
$\mathbf{7 5}$ & 0,246 & 0,307 & 0,334 & 0,345 & 0,350 & 0,357 & 0,359 & 0,362 & 0,365 & 0,368 & 0,370 & 0,374 & 0,375 & 0,376 \\
$\mathbf{1 0 0}$ & 0,190 & 0,246 & 0,295 & 0,324 & 0,338 & 0,345 & 0,350 & 0,354 & 0,359 & 0,359 & 0,362 & 0,363 & 0,366 & 0,368 \\
$\mathbf{1 2 5}$ & 0,160 & 0,199 & 0,246 & 0,284 & 0,315 & 0,332 & 0,339 & 0,345 & 0,349 & 0,351 & 0,356 & 0,359 & 0,359 & 0,361 \\
$\mathbf{1 5 0}$ & 0,128 & 0,175 & 0,207 & 0,246 & 0,277 & 0,307 & 0,324 & 0,334 & 0,340 & 0,345 & 0,348 & 0,350 & 0,354 & 0,357 \\
$\mathbf{1 7 5}$ & 0,051 & 0,156 & 0,183 & 0,212 & 0,246 & 0,272 & 0,302 & 0,317 & 0,331 & 0,337 & 0,341 & 0,345 & 0,348 & 0,350 \\
$\mathbf{2 0 0}$ & 0,012 & 0,128 & 0,164 & 0,190 & 0,217 & 0,246 & 0,270 & 0,295 & 0,313 & 0,324 & 0,333 & 0,338 & 0,341 & 0,345 \\
$\mathbf{2 2 5}$ & 0,001 & 0,071 & 0,151 & 0,175 & 0,194 & 0,223 & 0,246 & 0,266 & 0,289 & 0,307 & 0,318 & 0,329 & 0,334 & 0,338 \\
$\mathbf{2 5 0}$ & $\mathbf{0 , 0 0 0}$ & 0,023 & 0,128 & 0,160 & 0,181 & 0,199 & 0,224 & 0,246 & 0,266 & 0,284 & 0,304 & 0,315 & 0,324 & 0,332 \\
\hline
\end{tabular}




\begin{tabular}{|c|c|c|c|c|c|c|c|c|c|c|c|c|c|c|}
\hline 275 & 0,000 & 0,009 & 0,082 & 0,146 & 0,167 & 0,186 & 0,203 & 0,227 & 0,246 & 0,263 & 0,280 & 0,299 & 0,312 & 0,319 \\
\hline 300 & 0,000 & 0,001 & 0,041 & 0,128 & 0,158 & 0,175 & 0,190 & 0,207 & 0,229 & 0,246 & 0,263 & 0,277 & 0,295 & 0,307 \\
\hline 325 & 0,000 & 0,000 & 0,018 & 0,087 & 0,145 & 0,163 & 0,179 & 0,192 & 0,209 & 0,229 & 0,246 & 0,262 & 0,274 & 0,293 \\
\hline 350 & 0,000 & 0,000 & 0,006 & 0,051 & 0,128 & 0,156 & 0,168 & 0,183 & 0,195 & 0,212 & 0,230 & 0,246 & 0,261 & 0,272 \\
\hline 375 & 0,000 & 0,000 & 0,001 & 0,023 & 0,093 & 0,143 & 0,160 & 0,175 & 0,186 & 0,199 & 0,214 & 0,232 & 0,246 & 0,259 \\
\hline 400 & 0,000 & 0,000 & 0,000 & 0,012 & 0,061 & 0,128 & 0,152 & 0,164 & 0,179 & 0,190 & 0,201 & 0,217 & 0,233 & 0,246 \\
\hline 425 & 0,000 & 0,000 & 0,000 & 0,006 & 0,033 & 0,097 & 0,142 & 0,158 & 0,170 & 0,183 & 0,191 & 0,204 & 0,220 & 0,235 \\
\hline 450 & 0,000 & 0,000 & 0,000 & 0,001 & 0,020 & 0,071 & 0,128 & 0,151 & 0,163 & 0,175 & 0,184 & 0,194 & 0,207 & 0,223 \\
\hline 475 & 0,000 & 0,000 & 0,000 & 0,000 & 0,010 & 0,044 & 0,100 & 0,140 & 0,158 & 0,166 & 0,178 & 0,188 & 0,197 & 0,208 \\
\hline 500 & 0,000 & 0,000 & 0,000 & 0,000 & 0,005 & 0,023 & 0,076 & 0,128 & 0,148 & 0,160 & 0,171 & 0,181 & 0,190 & 0,199 \\
\hline 525 & 0,000 & 0,000 & 0,000 & 0,000 & 0,001 & 0,014 & 0,051 & 0,103 & 0,140 & 0,156 & 0,163 & 0,175 & 0,183 & 0,191 \\
\hline 550 & 0,000 & 0,000 & 0,000 & 0,000 & $\mathbf{0 , 0 0 0}$ & 0,009 & 0,032 & 0,082 & 0,128 & 0,146 & 0,159 & 0,167 & 0,178 & 0,186 \\
\hline 575 & 0,000 & 0,000 & 0,000 & 0,000 & 0,000 & 0,003 & 0,021 & 0,058 & 0,105 & 0,140 & 0,152 & 0,162 & 0,172 & 0,180 \\
\hline 600 & 0,000 & 0,000 & 0,000 & 0,000 & 0,000 & 0,001 & 0,012 & 0,041 & 0,084 & 0,128 & 0,145 & 0,158 & 0,164 & 0,175 \\
\hline 625 & 0,000 & 0,000 & 0,000 & 0,000 & 0,000 & 0,001 & 0,008 & 0,023 & 0,066 & 0,107 & 0,139 & 0,152 & 0,160 & 0,168 \\
\hline 650 & 0,000 & 0,000 & 0,000 & 0,000 & 0,000 & $\mathbf{0 , 0 0 0}$ & 0,003 & 0,018 & 0,046 & 0,087 & 0,128 & 0,145 & 0,158 & 0,163 \\
\hline 675 & 0,000 & 0,000 & 0,000 & 0,000 & 0,000 & 0,000 & 0,001 & 0,011 & 0,032 & 0,071 & 0,108 & 0,139 & 0,151 & 0,159 \\
\hline 700 & 0,000 & 0,000 & 0,000 & 0,000 & 0,000 & 0,000 & 0,001 & 0,006 & 0,021 & 0,051 & 0,088 & 0,128 & 0,144 & 0,156 \\
\hline 725 & 0,000 & 0,000 & 0,000 & 0,000 & 0,000 & 0,000 & 0,000 & 0,003 & 0,013 & 0,037 & 0,075 & 0,109 & 0,138 & 0,148 \\
\hline 750 & 0,000 & 0,000 & 0,000 & 0,000 & 0,000 & 0,000 & 0,000 & 0,001 & 0,010 & 0,023 & 0,056 & 0,093 & 0,128 & 0,143 \\
\hline 775 & 0,000 & 0,000 & 0,000 & 0,000 & 0,000 & 0,000 & 0,000 & 0,001 & 0,006 & 0,018 & 0,043 & 0,078 & 0,113 & 0,137 \\
\hline 800 & 0,000 & 0,000 & 0,000 & 0,000 & 0,000 & 0,000 & 0,000 & $\mathbf{0 , 0 0 0}$ & 0,003 & 0,012 & 0,031 & 0,061 & 0,095 & 0,128 \\
\hline 825 & 0,000 & 0,000 & 0,000 & 0,000 & 0,000 & 0,000 & 0,000 & 0,000 & 0,001 & 0,009 & 0,022 & 0,048 & 0,082 & 0,114 \\
\hline 850 & 0,000 & 0,000 & 0,000 & 0,000 & 0,000 & 0,000 & 0,000 & 0,000 & 0,001 & 0,006 & 0,015 & 0,033 & 0,067 & 0,097 \\
\hline 875 & 0,000 & 0,000 & 0,000 & 0,000 & 0,000 & 0,000 & 0,000 & 0,000 & $\mathbf{0 , 0 0 0}$ & 0,002 & 0,011 & 0,023 & 0,051 & 0,083 \\
\hline 900 & 0,000 & 0,000 & 0,000 & 0,000 & 0,000 & 0,000 & 0,000 & 0,000 & 0,000 & 0,001 & 0,008 & 0,020 & 0,041 & 0,071 \\
\hline 925 & 0,000 & 0,000 & 0,000 & 0,000 & 0,000 & 0,000 & 0,000 & 0,000 & 0,000 & 0,001 & 0,005 & 0,013 & 0,031 & 0,056 \\
\hline 950 & 0,000 & 0,000 & 0,000 & 0,000 & 0,000 & 0,000 & 0,000 & 0,000 & 0,000 & $\mathbf{0 , 0 0 0}$ & 0,002 & 0,010 & 0,022 & 0,044 \\
\hline 975 & 0,000 & 0,000 & 0,000 & 0,000 & 0,000 & 0,000 & 0,000 & 0,000 & 0,000 & 0,000 & 0,001 & 0,008 & 0,018 & 0,033 \\
\hline 1000 & 0,000 & 0,000 & 0,000 & 0,000 & 0,000 & 0,000 & 0,000 & 0,000 & 0,000 & 0,000 & $\mathbf{0 , 0 0 1}$ & 0,005 & 0,012 & $\mathbf{0 , 0 2 3}$ \\
\hline
\end{tabular}

Table 9. Annual probability of defaults for different levels of mortgage loans and rents with the maturity of eighteen years

\begin{tabular}{ccccccccccccccc}
\hline & $\mathbf{7 5 0 0 0}$ & $\mathbf{1 0 0 0 0 0}$ & $\mathbf{1 2 5 0 0 0}$ & $\mathbf{1 5 0 0 0 0}$ & $\mathbf{1 7 5 0 0 0}$ & $\mathbf{2 0 0 0 0 0}$ & $\mathbf{2 2 5 0 0 0}$ & $\mathbf{2 5 0 0 0 0}$ & $\mathbf{2 7 5 0 0 0}$ & $\mathbf{3 0 0 0 0 0}$ & $\mathbf{3 2 5 0 0 0}$ & $\mathbf{3 5 0 0 0 0}$ & $\mathbf{3 7 5 0 0 0}$ & $\mathbf{4 0 0 0 0 0}$ \\
\hline $\mathbf{0}$ & 0,395 & 0,395 & 0,395 & 0,395 & 0,395 & 0,395 & 0,395 & 0,395 & 0,395 & 0,395 & 0,395 & 0,395 & 0,395 & 0,395 \\
$\mathbf{2 5}$ & 0,352 & 0,361 & 0,366 & 0,370 & 0,374 & 0,376 & 0,379 & 0,380 & 0,381 & 0,382 & 0,382 & 0,382 & 0,383 & 0,383 \\
$\mathbf{5 0}$ & 0,307 & 0,337 & 0,346 & 0,352 & 0,359 & 0,361 & 0,362 & 0,366 & 0,368 & 0,370 & 0,374 & 0,374 & 0,376 & 0,376 \\
$\mathbf{7 5}$ & 0,225 & 0,279 & 0,319 & 0,337 & 0,345 & 0,349 & 0,352 & 0,358 & 0,359 & 0,361 & 0,362 & 0,364 & 0,366 & 0,368 \\
$\mathbf{1 0 0}$ & 0,178 & 0,225 & 0,267 & 0,307 & 0,326 & 0,337 & 0,343 & 0,346 & 0,350 & 0,352 & 0,357 & 0,359 & 0,359 & 0,361 \\
$\mathbf{1 2 5}$ & 0,148 & 0,186 & 0,225 & 0,260 & 0,293 & 0,315 & 0,329 & 0,337 & 0,341 & 0,345 & 0,348 & 0,350 & 0,352 & 0,356 \\
$\mathbf{1 5 0}$ & 0,087 & 0,163 & 0,190 & 0,225 & 0,252 & 0,279 & 0,307 & 0,319 & 0,332 & 0,337 & 0,340 & 0,345 & 0,346 & 0,349 \\
$\mathbf{1 7 5}$ & 0,023 & 0,140 & 0,172 & 0,194 & 0,225 & 0,250 & 0,272 & 0,297 & 0,313 & 0,323 & 0,332 & 0,337 & 0,340 & 0,344 \\
$\mathbf{2 0 0}$ & 0,005 & 0,087 & 0,157 & 0,178 & 0,198 & 0,225 & 0,248 & 0,267 & 0,288 & 0,307 & 0,316 & 0,326 & 0,333 & 0,337 \\
$\mathbf{2 2 5}$ & $\mathbf{0 , 0 0 0}$ & 0,034 & 0,135 & 0,163 & 0,183 & 0,201 & 0,225 & 0,245 & 0,263 & 0,279 & 0,299 & 0,312 & 0,319 & 0,328 \\
$\mathbf{2 5 0}$ & 0,000 & 0,011 & 0,087 & 0,148 & 0,168 & 0,186 & 0,201 & 0,225 & 0,240 & 0,260 & 0,274 & 0,293 & 0,307 & 0,315 \\
$\mathbf{2 7 5}$ & 0,000 & 0,002 & 0,046 & 0,130 & 0,159 & 0,174 & 0,189 & 0,204 & 0,225 & 0,239 & 0,256 & 0,271 & 0,287 & 0,301 \\
$\mathbf{3 0 0}$ & 0,000 & $\mathbf{0 , 0 0 0}$ & 0,019 & 0,087 & 0,144 & 0,163 & 0,178 & 0,190 & 0,206 & 0,225 & 0,239 & 0,252 & 0,267 & 0,279 \\
$\mathbf{3 2 5}$ & 0,000 & 0,000 & 0,008 & 0,052 & 0,126 & 0,153 & 0,167 & 0,181 & 0,191 & 0,207 & 0,225 & 0,238 & 0,250 & 0,264 \\
$\mathbf{3 5 0}$ & 0,000 & 0,000 & 0,001 & 0,023 & 0,087 & 0,140 & 0,159 & 0,172 & 0,183 & 0,194 & 0,207 & 0,225 & 0,238 & 0,250 \\
$\mathbf{3 7 5}$ & 0,000 & 0,000 & $\mathbf{0 , 0 0 0}$ & 0,011 & 0,056 & 0,122 & 0,148 & 0,163 & 0,176 & 0,186 & 0,195 & 0,208 & 0,225 & 0,238 \\
$\mathbf{4 0 0}$ & 0,000 & 0,000 & 0,000 & 0,005 & 0,031 & 0,087 & 0,139 & 0,157 & 0,166 & 0,178 & 0,188 & 0,198 & 0,210 & 0,225 \\
$\mathbf{4 2 5}$ & 0,000 & 0,000 & 0,000 & 0,001 & 0,017 & 0,060 & 0,120 & 0,145 & 0,159 & 0,170 & 0,181 & 0,190 & 0,199 & 0,211 \\
\hline & & & & & & & & & & & & &
\end{tabular}




\begin{tabular}{|c|c|c|c|c|c|c|c|c|c|c|c|c|c|c|}
\hline 450 & 0,000 & 0,000 & 0,000 & $\mathbf{0 , 0 0 0}$ & 0,009 & 0,034 & 0,087 & 0,135 & 0,153 & 0,163 & 0,173 & 0,183 & 0,190 & 0,201 \\
\hline 475 & 0,000 & 0,000 & 0,000 & 0,000 & 0,002 & 0,021 & 0,064 & 0,116 & 0,143 & 0,158 & 0,164 & 0,177 & 0,183 & 0,191 \\
\hline 500 & 0,000 & 0,000 & 0,000 & 0,000 & 0,001 & 0,011 & 0,042 & 0,087 & 0,133 & 0,148 & 0,160 & 0,168 & 0,178 & 0,186 \\
\hline 525 & 0,000 & 0,000 & 0,000 & 0,000 & 0,000 & 0,006 & 0,023 & 0,067 & 0,114 & 0,140 & 0,155 & 0,163 & 0,172 & 0,180 \\
\hline 550 & 0,000 & 0,000 & 0,000 & 0,000 & 0,000 & 0,002 & 0,014 & 0,046 & 0,087 & 0,130 & 0,146 & 0,159 & 0,163 & 0,174 \\
\hline 575 & 0,000 & 0,000 & 0,000 & 0,000 & 0,000 & 0,001 & 0,009 & 0,029 & 0,071 & 0,109 & 0,139 & 0,152 & 0,160 & 0,168 \\
\hline 600 & 0,000 & 0,000 & 0,000 & 0,000 & 0,000 & 0,000 & 0,005 & 0,019 & 0,049 & 0,087 & 0,128 & 0,144 & 0,157 & 0,163 \\
\hline 625 & 0,000 & 0,000 & 0,000 & 0,000 & 0,000 & 0,000 & 0,002 & 0,011 & 0,032 & 0,073 & 0,107 & 0,137 & 0,148 & 0,159 \\
\hline 650 & 0,000 & 0,000 & 0,000 & 0,000 & 0,000 & 0,000 & 0,001 & 0,008 & 0,022 & 0,052 & 0,087 & 0,126 & 0,142 & 0,153 \\
\hline 675 & 0,000 & 0,000 & 0,000 & 0,000 & 0,000 & 0,000 & 0,000 & 0,003 & 0,013 & 0,034 & 0,073 & 0,107 & 0,135 & 0,146 \\
\hline 700 & 0,000 & 0,000 & 0,000 & 0,000 & 0,000 & 0,000 & 0,000 & 0,001 & 0,010 & 0,023 & 0,053 & 0,087 & 0,124 & 0,140 \\
\hline 725 & 0,000 & 0,000 & 0,000 & 0,000 & 0,000 & 0,000 & 0,000 & 0,001 & & 0,017 & & 0,074 & 0,106 & 0,134 \\
\hline 750 & 0,000 & 0,000 & 0,000 & 0,000 & 0,000 & 0,000 & 0,000 & 0,000 & 0,002 & 0,011 & 0,027 & 0,056 & 0,087 & 0,122 \\
\hline 775 & 0,000 & 0,000 & 0,000 & 0,000 & 0,000 & 0,000 & 0,000 & 0,000 & 0,001 & 0,008 & 0,021 & 0,043 & 0,075 & 0,105 \\
\hline 800 & 0,000 & 0,000 & 0,000 & 0,000 & 0,000 & 0,000 & 0,000 & 0,000 & 0,001 & 0,005 & 0,013 & 0,031 & 0,058 & 0,087 \\
\hline 825 & 0,000 & 0,000 & 0,000 & 0,000 & 0,000 & 0,000 & 0,000 & 0,000 & 0,000 & 0,002 & 0,010 & 0,022 & 0,046 & 0,076 \\
\hline 850 & 0,000 & 0,000 & 0,000 & 0,000 & 0,000 & 0,000 & 0,000 & 0,000 & 0,000 & 0,001 & 0,006 & 0,017 & 0,033 & 0,060 \\
\hline 875 & 0,000 & 0,000 & 0,000 & 0,000 & 0,000 & 0,000 & 0,000 & 0,000 & 0,000 & 0,001 & 0,003 & 0,011 & 0,023 & 0,048 \\
\hline 900 & 0,000 & 0,000 & 0,000 & 0,000 & 0,000 & 0,000 & 0,000 & 0,000 & 0,000 & $\mathbf{0 , 0 0 0}$ & 0,002 & 0,009 & 0,019 & 0,034 \\
\hline 925 & 0,000 & 0,000 & 0,000 & 0,000 & 0,000 & 0,000 & 0,000 & 0,000 & 0,000 & 0,000 & 0,001 & 0,006 & 0,013 & 0,026 \\
\hline 950 & 0,000 & 0,000 & 0,000 & 0,000 & 0,000 & 0,000 & 0,000 & 0,000 & 0,000 & 0,000 & 0,000 & 0,002 & 0,010 & 0,021 \\
\hline 975 & 0,000 & 0,000 & 0,000 & 0,000 & 0,000 & 0,000 & 0,000 & 0,000 & 0,000 & 0,000 & 0,000 & 0,001 & 0,008 & 0,014 \\
\hline 1000 & 0,000 & 0,000 & 0,000 & 0,000 & 0,000 & 0,000 & 0,000 & 0,000 & 0,000 & 0,000 & 0,000 & 0,001 & 0,005 & 0,011 \\
\hline
\end{tabular}

Table 10. Annual probability of defaults for different levels of mortgage loans and rents with the maturity of nineteen years

\begin{tabular}{|c|c|c|c|c|c|c|c|c|c|c|c|c|c|c|}
\hline & 75000 & 00000 & 125000 & 150000 & 175000 & 200000 & 225000 & 250000 & 275000 & 300000 & 325000 & 350000 & 375000 & 400000 \\
\hline 0 & 0,387 & 0,387 & 0,387 & 0,387 & 0,387 & 0,387 & 0,387 & 0,387 & 0,387 & 0,387 & 0,387 & 0,387 & 0,387 & 0,387 \\
\hline 25 & 0,346 & 0,356 & 0,359 & 0 & 365 & 0,368 & 370 & 0,373 & 0,374 & 375 & 0,376 & 0,376 & 0,377 & 0,377 \\
\hline 50 & 0,277 & 0,323 & 0,338 & 0,346 & 0,350 & 0,356 & 0,359 & 0,359 & 0,361 & 0,362 & 0,363 & 0,365 & 0,368 & 0,368 \\
\hline 75 & 0,201 & 0,254 & 0,300 & 0,323 & 0,335 & 0,342 & 0,346 & 0,350 & 0,351 & 0,356 & 0,358 & 0,359 & 0,359 & 0,361 \\
\hline 100 & 0,164 & 0,201 & 0,245 & 0,277 & 0,307 & 0,323 & 0,333 & 0,338 & 0,344 & 0,346 & 0,349 & 0,350 & 0,352 & 0,356 \\
\hline 125 & 0,133 & 0,174 & 0,201 & 0,238 & 265 & 0,293 & 0,312 & 0,323 & 0,332 & 0,337 & 0,340 & 0,344 & 0,346 & 0,348 \\
\hline 150 & 0,052 & 0,152 & 0,179 & 0,201 & 0,232 & 0,254 & 0,277 & 0,300 & 0,314 & 0,323 & 0,332 & 0,335 & 0,338 & 0,342 \\
\hline 175 & 0,011 & 0,115 & 0,160 & 0,183 & 0,201 & 0,229 & 0,250 & 0,269 & 0,288 & 0,306 & 0,315 & 0,323 & 0,331 & 0,333 \\
\hline 200 & 0,001 & 0,052 & 0,142 & 0,164 & 0,183 & 0,201 & 0,226 & 0,245 & 0,263 & 0,277 & 0,295 & 0,307 & 0,315 & 0,323 \\
\hline 225 & 0,000 & 0,017 & 0,101 & 0,152 & 0,170 & 0,186 & 0,201 & 0,223 & 0,239 & 0,254 & 0,271 & 0,285 & 0,300 & 0,310 \\
\hline 250 & 0,000 & 0,003 & 0,052 & 0,133 & 0,159 & 0,174 & 0,188 & 0,201 & 0,222 & 0,238 & 0,250 & 0,265 & 0,277 & 0,293 \\
\hline 275 & 0,000 & 0,000 & 0,021 & 0,091 & 0,145 & 0,161 & 0,178 & 0,189 & 0,201 & 0,219 & 0,236 & 0,248 & 0,261 & 0,272 \\
\hline 300 & 0,000 & 0,000 & 0,008 & 0,052 & 0,125 & 0,152 & 0,164 & 0,179 & 0,190 & 0,201 & 0,217 & 0,232 & 0,245 & 0,254 \\
\hline 325 & 0,000 & 0,000 & 0,001 & 0,023 & 0,084 & 0,139 & 0,159 & 0,168 & 0,181 & 0,190 & 0,201 & 0,215 & 0,229 & 0,239 \\
\hline 350 & 0,000 & 0,000 & & & 0,052 & & 0,145 & 0,160 & & & & 0,201 & 0,213 & 0,229 \\
\hline 375 & 0,000 & 0,000 & 0,000 & 0,003 & 0,026 & 0,081 & 0,133 & 0,152 & 0,163 & & 0,183 & 0,191 & 0,201 & 0,213 \\
\hline 400 & & & & & & & & & & & & & & 0,201 \\
\hline 425 & 0,000 & 0,000 & 0,000 & $\mathbf{0 , 0 0 0}$ & 0,006 & 0,029 & 0,079 & 0,128 & 0,146 & & 0,168 & 0,178 & 0,185 & 0,192 \\
\hline 450 & 0,000 & 0,000 & 0,000 & 0,000 & 0,002 & 0,017 & 0,052 & 0,101 & 0,138 & 0,152 & 0,161 & 0,170 & 0,179 & 0,186 \\
\hline 475 & 0,000 & 0,000 & 0,000 & 0,000 & $\mathbf{0 , 0 0 0}$ & 0,009 & 0,032 & 0,076 & 0,122 & 0,143 & & 0,163 & 0,172 & 0,180 \\
\hline 500 & 0,000 & 0,000 & 0,000 & 0,000 & 0,000 & 0,003 & 0,020 & 0,052 & 0,095 & 0,133 & 0,147 & 0,159 & 0,164 & 0,174 \\
\hline 525 & 0,000 & 0,000 & 0,000 & 0,000 & 0,000 & 0,001 & 0,011 & 0,033 & 0,075 & 0,115 & 0,140 & 0,152 & 0,160 & 0,167 \\
\hline 550 & 0,000 & 0,000 & 0,000 & 0,000 & 0,000 & 0,000 & 0,006 & 0,021 & 0,052 & 0,091 & 0,129 & 0,145 & 0,157 & 0,161 \\
\hline 575 & 0,000 & 0,000 & 0,000 & 0,000 & 0,000 & 0,000 & 0,002 & 0,012 & 0,033 & 0,073 & 0,107 & 0,137 & 0,148 & 0,159 \\
\hline 600 & 0,000 & 0,000 & 0,000 & 0,000 & 0,000 & 0,000 & 0,001 & 0,008 & 0,022 & 0,052 & 0,087 & 0,125 & 0,142 & 0,152 \\
\hline
\end{tabular}




\begin{tabular}{ccccccccccccccc}
\hline $\mathbf{6 2 5}$ & 0,000 & 0,000 & 0,000 & 0,000 & 0,000 & 0,000 & $\mathbf{0 , 0 0 0}$ & 0,003 & 0,014 & 0,034 & 0,073 & 0,105 & 0,133 & 0,145 \\
$\mathbf{6 5 0}$ & 0,000 & 0,000 & 0,000 & 0,000 & 0,000 & 0,000 & 0,000 & 0,001 & 0,010 & 0,023 & 0,052 & 0,084 & 0,121 & 0,139 \\
$\mathbf{6 7 5}$ & 0,000 & 0,000 & 0,000 & 0,000 & 0,000 & 0,000 & 0,000 & 0,001 & 0,005 & 0,017 & 0,037 & 0,070 & 0,101 & 0,130 \\
$\mathbf{7 0 0}$ & 0,000 & 0,000 & 0,000 & 0,000 & 0,000 & 0,000 & 0,000 & $\mathbf{0 , 0 0 0}$ & 0,002 & 0,011 & 0,025 & 0,052 & 0,084 & 0,115 \\
$\mathbf{7 2 5}$ & 0,000 & 0,000 & 0,000 & 0,000 & 0,000 & 0,000 & 0,000 & 0,000 & 0,001 & 0,008 & 0,018 & 0,039 & 0,069 & 0,097 \\
$\mathbf{7 5 0}$ & 0,000 & 0,000 & 0,000 & 0,000 & 0,000 & 0,000 & 0,000 & 0,000 & $\mathbf{0 , 0 0 0}$ & 0,003 & 0,012 & 0,026 & 0,052 & 0,081 \\
$\mathbf{7 7 5}$ & 0,000 & 0,000 & 0,000 & 0,000 & 0,000 & 0,000 & 0,000 & 0,000 & 0,000 & 0,001 & 0,008 & 0,021 & 0,040 & 0,067 \\
$\mathbf{8 0 0}$ & 0,000 & 0,000 & 0,000 & 0,000 & 0,000 & 0,000 & 0,000 & 0,000 & 0,000 & 0,001 & 0,005 & 0,013 & 0,029 & 0,052 \\
$\mathbf{8 2 5}$ & 0,000 & 0,000 & 0,000 & 0,000 & 0,000 & 0,000 & 0,000 & 0,000 & 0,000 & $\mathbf{0 , 0 0 0}$ & 0,002 & 0,010 & 0,021 & 0,040 \\
$\mathbf{8 5 0}$ & 0,000 & 0,000 & 0,000 & 0,000 & 0,000 & 0,000 & 0,000 & 0,000 & 0,000 & 0,000 & 0,001 & 0,006 & 0,014 & 0,029 \\
$\mathbf{8 7 5}$ & 0,000 & 0,000 & 0,000 & 0,000 & 0,000 & 0,000 & 0,000 & 0,000 & 0,000 & 0,000 & 0,001 & 0,003 & 0,011 & 0,022 \\
$\mathbf{9 0 0}$ & 0,000 & 0,000 & 0,000 & 0,000 & 0,000 & 0,000 & 0,000 & 0,000 & 0,000 & 0,000 & $\mathbf{0 , 0 0 0}$ & 0,002 & 0,008 & 0,017 \\
$\mathbf{9 2 5}$ & 0,000 & 0,000 & 0,000 & 0,000 & 0,000 & 0,000 & 0,000 & 0,000 & 0,000 & 0,000 & 0,000 & 0,001 & 0,005 & 0,011 \\
$\mathbf{9 5 0}$ & 0,000 & 0,000 & 0,000 & 0,000 & 0,000 & 0,000 & 0,000 & 0,000 & 0,000 & 0,000 & 0,000 & $\mathbf{0 , 0 0 0}$ & 0,002 & 0,009 \\
$\mathbf{9 7 5}$ & 0,000 & 0,000 & 0,000 & 0,000 & 0,000 & 0,000 & 0,000 & 0,000 & 0,000 & 0,000 & 0,000 & 0,000 & 0,001 & 0,006 \\
$\mathbf{1 0 0 0}$ & 0,000 & 0,000 & 0,000 & 0,000 & 0,000 & 0,000 & 0,000 & 0,000 & 0,000 & 0,000 & 0,000 & 0,000 & $\mathbf{0 , 0 0 1}$ & $\mathbf{0 , 0 0 3}$ \\
\hline
\end{tabular}

Table 11. Probability of defaults for different levels of mortgage loans and rents with the maturity of twenty years

\begin{tabular}{|c|c|c|c|c|c|c|c|c|c|c|c|c|c|c|}
\hline & 75000 & 00000 & 125000 & 150000 & 175000 & 200000 & 225000 & 250000 & 275000 & 300000 & 325000 & 350000 & 375000 & 400000 \\
\hline 0 & 0,380 & 0,380 & 0,380 & 0,380 & 0,380 & 0,380 & 0,380 & 0,380 & 0,380 & 0,380 & 0,380 & 0,380 & 0,380 & 0,380 \\
\hline 25 & ,338 & 0,347 & 352 & 358 & 359 & 0,361 & 0,362 & 0,362 & 0,364 & 0,366 & 0,367 & ,368 & ,368 & ,369 \\
\hline 50 & 0,251 & 0,305 & 0,328 & 0,338 & 0,344 & 0,347 & 0,350 & 0,352 & 0,356 & 0,358 & 0,359 & 0,359 & 0,359 & 0,361 \\
\hline 75 & 186 & 0,2 & & & & & & 0,342 & 345 & 0,347 & & & & \\
\hline 100 & 156 & 0,186 & & & & & 0,317 & & & 0,338 & 0,341 & & & 0,347 \\
\hline 125 & 0,095 & 0,161 & & & 0,239 & 0,264 & 0,284 & 0,305 & 0,315 & 0,324 & 0,332 & 0,334 & 0,338 & 0,340 \\
\hline 150 & 0,023 & 0,137 & & & 0,207 & 0,234 & 0,251 & 0,271 & 0,288 & 0,305 & 0,314 & 0,320 & 0,328 & 0,333 \\
\hline 175 & 0,002 & 0,075 & & 0,168 & 0,186 & 0,205 & 0,228 & 0,245 & 0,261 & 0,274 & 0,293 & 0,305 & 0,313 & 0,318 \\
\hline 200 & 0,000 & 0,023 & & 0,156 & 0,172 & 0,186 & 0,201 & 0,223 & 0,238 & 0,251 & 0,266 & 0,278 & & 0,305 \\
\hline 225 & 0,000 & 0,006 & 0,062 & 0,137 & 0,159 & 0,174 & 0,186 & 0,200 & 0,217 & 0,234 & 0,246 & 0,259 & 0,271 & 0,281 \\
\hline 250 & 0,000 & 0,001 & 0,023 & 0,095 & 0,145 & 0,161 & 0,177 & 0,186 & 0,199 & 0,213 & 0,229 & 0,239 & 0,251 & 0,264 \\
\hline 275 & 0,000 & 0,000 & 0,009 & 0,053 & 0,125 & 0,151 & 0,163 & 0,178 & 0,186 & 0,197 & 0,210 & 0,226 & 0,238 & 0,248 \\
\hline 300 & 0,000 & 0,000 & 0,001 & 0,023 & 0,083 & 0,137 & 0,156 & 0,164 & 0,178 & 0,186 & 0,196 & 0,207 & 0,223 & 0,234 \\
\hline 325 & 0,000 & 0,000 & 0,000 & 0,011 & 0,049 & 0,107 & 0,143 & 0,159 & 0,168 & 0,179 & 0,186 & 0,195 & 0,207 & 0,219 \\
\hline 350 & 0,000 & 0,000 & 0,000 & 0,002 & 0,023 & 0,075 & 0,128 & 0,146 & 0,159 & 0,168 & 0,179 & 0,186 & 0,194 & 0,205 \\
\hline 375 & 0,000 & 0,000 & 0,000 & 0,001 & 0,011 & 0,044 & 0,095 & 0,137 & 0,152 & 0,161 & 0,170 & 0,180 & 0,186 & 0,193 \\
\hline 400 & 0,000 & 0,000 & 0,000 & 0,000 & 0,005 & 0,023 & 0,069 & 0,116 & 0,143 & 0,156 & 0,163 & 0,172 & 0,181 & 0,186 \\
\hline 425 & 0,000 & 0,000 & 0,000 & 0,000 & 0,001 & 0,012 & 0,042 & 0,084 & 0,130 & 0,145 & 0,159 & 0,163 & 0,173 & 0,181 \\
\hline 450 & 0,000 & 0,000 & 0,000 & 0,000 & 0,000 & 0,006 & 0,023 & 0,062 & 0,105 & 0,137 & 0,150 & 0,159 & 0,164 & 0,174 \\
\hline 475 & 0,000 & 0,000 & 0,000 & 0,000 & 0,000 & 0,002 & 0,013 & 0,039 & 0,080 & 0,122 & 0,142 & 0,153 & 0,160 & 0,166 \\
\hline 500 & 0,000 & 0,000 & 0,000 & 0,000 & 0,000 & 0,001 & 0,008 & 0,023 & 0,055 & 0,095 & 0,131 & 0,145 & 0,156 & 0,161 \\
\hline 525 & 0,000 & 0,000 & 0,000 & 0,000 & 0,000 & 0,000 & 0,002 & 0,014 & 0,038 & 0,075 & 0,111 & 0,137 & 0,146 & 0,158 \\
\hline 550 & 0,000 & 0,000 & 0,000 & 0,000 & 0,000 & 0,000 & 0,001 & 0,009 & 0,023 & 0,053 & 0,087 & 0,125 & 0,140 & 0,151 \\
\hline 575 & 0,000 & 0,000 & 0,000 & & 0,000 & 0,000 & 0,000 & 0,004 & 0,014 & 0,035 & 0,071 & 0,102 & 0,132 & 0,144 \\
\hline 600 & 0,000 & 0,000 & 0,000 & 0,000 & 0,000 & 0,000 & 0,000 & 0,001 & 0,010 & 0,023 & 0,050 & 0,083 & 0,116 & 0,137 \\
\hline 625 & 0,000 & 0,000 & 0,000 & 0,000 & 0,000 & 0,000 & 0,000 & 0,001 & 0,005 & 0,015 & 0,033 & 0,067 & 0,095 & 0,126 \\
\hline 650 & 0,000 & 0,000 & 0,000 & 0,000 & 0,000 & 0,000 & 0,000 & 0,000 & 0,002 & 0,011 & 0,023 & 0,049 & 0,079 & 0,107 \\
\hline 675 & 0,000 & 0,000 & 0,000 & 0,000 & 0,000 & 0,000 & 0,000 & 0,000 & 0,001 & 0,006 & 0,016 & 0,033 & 0,062 & 0,088 \\
\hline 700 & 0,000 & 0,000 & 0,000 & 0,000 & 0,000 & 0,000 & 0,000 & 0,000 & 0,000 & 0,002 & 0,011 & 0,023 & 0,045 & 0,075 \\
\hline 725 & 0,000 & 0,000 & 0,000 & 0,000 & 0,000 & 0,000 & 0,000 & 0,000 & 0,000 & 0,001 & 0,008 & 0,016 & 0,032 & 0,057 \\
\hline 750 & 0,000 & 0,000 & 0,000 & 0,000 & 0,000 & 0,000 & 0,000 & 0,000 & 0,000 & 0,001 & 0,003 & 0,011 & 0,023 & 0,044 \\
\hline 775 & 0,000 & 0,000 & 0,000 & 0,000 & 0,000 & 0,000 & 0,000 & 0,000 & 0,000 & 0,000 & 0,001 & 0,008 & 0,017 & 0,032 \\
\hline
\end{tabular}




\begin{tabular}{llllllllllllllll}
\hline $\mathbf{8 0 0}$ & 0,000 & 0,000 & 0,000 & 0,000 & 0,000 & 0,000 & 0,000 & 0,000 & 0,000 & 0,000 & 0,001 & 0,005 & 0,012 & 0,023 \\
$\mathbf{8 2 5}$ & 0,000 & 0,000 & 0,000 & 0,000 & 0,000 & 0,000 & 0,000 & 0,000 & 0,000 & 0,000 & 0,000 & 0,002 & 0,009 & 0,018 \\
$\mathbf{8 5 0}$ & 0,000 & 0,000 & 0,000 & 0,000 & 0,000 & 0,000 & 0,000 & 0,000 & 0,000 & 0,000 & 0,000 & 0,001 & 0,005 & 0,012 \\
$\mathbf{8 7 5}$ & 0,000 & 0,000 & 0,000 & 0,000 & 0,000 & 0,000 & 0,000 & 0,000 & 0,000 & 0,000 & 0,000 & 0,001 & 0,002 & 0,010 \\
$\mathbf{9 0 0}$ & 0,000 & 0,000 & 0,000 & 0,000 & 0,000 & 0,000 & 0,000 & 0,000 & 0,000 & 0,000 & 0,000 & 0,000 & 0,001 & 0,006 \\
$\mathbf{9 2 5}$ & 0,000 & 0,000 & 0,000 & 0,000 & 0,000 & 0,000 & 0,000 & 0,000 & 0,000 & 0,000 & 0,000 & 0,000 & 0,001 & 0,003 \\
$\mathbf{9 5 0}$ & 0,000 & 0,000 & 0,000 & 0,000 & 0,000 & 0,000 & 0,000 & 0,000 & 0,000 & 0,000 & 0,000 & 0,000 & 0,000 & 0,002 \\
$\mathbf{9 7 5}$ & 0,000 & 0,000 & 0,000 & 0,000 & 0,000 & 0,000 & 0,000 & 0,000 & 0,000 & 0,000 & 0,000 & 0,000 & 0,000 & 0,001 \\
$\mathbf{1 0 0 0}$ & 0,000 & 0,000 & 0,000 & 0,000 & 0,000 & 0,000 & 0,000 & 0,000 & 0,000 & 0,000 & 0,000 & 0,000 & 0,000 & 0,001 \\
\hline
\end{tabular}

\section{Copyrights}

Copyright for this article is retained by the author(s), with first publication rights granted to the journal.

This is an open-access article distributed under the terms and conditions of the Creative Commons Attribution license (http://creativecommons.org/licenses/by/4.0/). 